\title{
Disciplinary integration of digital games for science learning
}

\author{
Douglas B Clark ${ }^{1 *}$, Pratim Sengupta ${ }^{1}$, Corey E Brady ${ }^{2}$, Mario M Martinez-Garza ${ }^{1}$ and Stephen S Killingsworth ${ }^{1}$
}

\begin{abstract}
Background: In this paper, we investigate the relationship between theory and design in the context of creating digital games to support children's development of scientific expertise.

Synthesis: Theoretically, we consider two frameworks: Knowledge in Pieces (or KiP) and Science as Practice (or SaP). While KiP is a theory about the structure of human knowledge, SaP is a theoretical perspective about the development of scientific expertise that emphasizes the deeply intertwined nature of conceptual development and the development of epistemic and representational practices. We then synthesize research on modeling and games from our research group and others to create a composite argument and theoretical framing for the importance of disciplinary integration in the design of digital games for science learning.

Conclusions: We show how shifting from KiP to SaP as the underlying theoretical anchor in our designs results in shifting from focusing on conceptual integration to focusing on disciplinary integration. We first present our initial framing and design around conceptual integration. We then trace the evolution and rationale for disciplinary integration across our development and research on four game platforms. Finally, we discuss the implications and generalizability of disciplinary integration across the design of digital games for science learning.
\end{abstract}

Keywords: Digital games; Learning; Science education; Learning technologies

\section{Background}

Digital games provide a promising medium for science education (Clark et al. 2009; Honey and Hilton 2010; National Research Council 2009). The potential of digital games as a genre of educational technologies for fostering scientific expertise has been noted not only by educators but also by scientists (Federation of American Scientists 2006; Hines et al. 2009). There is now a growing body of research that shows that digital games can indeed be productively used to support science learning in K-12 classrooms (Clark et al. 2014a; Martinez-Garza et al. 2013; Wouters et al. 2013).

In this paper, we focus on theorizing the design of digital games to support the learning of core scientific concepts and representational practices. Theoretically, we consider two frameworks: Knowledge in Pieces (or KiP) (diSessa 1993; Hammer 1996; Sherin 2001; Clark et al. 2009) and Science as Practice (or SaP)

\footnotetext{
* Correspondence: doug.clark@vanderbilt.edu

${ }^{1}$ College of Education, Vanderbilt University, Box 230, 230 Appleton Place, Nashville, Tennessee 37203-5721, USA

Full list of author information is available at the end of the article
}

(Pickering 1995; Lehrer and Schauble 2006a; Duschl et al. 2007). While KiP is a theory about the structure of human knowledge, $\mathrm{SaP}$ is a theoretical perspective about the development of scientific expertise. Grounded in the history of science, SaP argues that the development of scientific concepts is deeply intertwined with the development of epistemic and representational practices (e.g., modeling). We report how these theoretical frameworks have shaped the design of our digital games for learning Newtonian dynamics across an extended design experiment. We show how shifting from KiP to SaP as the underlying theoretical anchor has enabled a shift from designing games that focus on conceptual integration (Clark and Martinez-Garza 2012) to games that focus on disciplinary integration. Whereas conceptually integrated games integrate the targeted conceptual relationships directly into the mechanics of the core game environment, disciplinary integration extends conceptual integration by incorporating disciplinary practices as well as conceptual relationships into the mechanics of interacting with, manipulating, or navigating the core game environment. 


\section{Knowledge in pieces as the theoretical anchor for conceptual integration}

The Knowledge-in-Pieces perspective frames conceptual change as a gradual process that relies on bootstrapping ideas that students bring to the instructional setting (diSessa 1988, 1993; Hammer 1996; Smith et al. 1993; Amin 2009; Jeppsson et al. 2013). In this perspective, human knowledge consists of many, loosely organized, fragmented pieces of knowledge (Clark 2006; diSessa 1993; Hammer et al. 2005). In framing this perspective, diSessa (1993) postulated that the building blocks of understanding are phenomenological primitives (p-prims). P-prims are small knowledge elements whose origins stem from repeated abstractions of familiar events. Cued upon recognition of contextual features, p-prims are used to construct intuitive understandings of the physical world. According to this perspective, naïve understanding is highly sensitive to context, with predictions and explanations depending in subtle ways on the particular knowledge elements that are triggered in particular reasoning situations.

According to the Knowledge-in-Pieces perspective, conceptual change involves a gradual development of coherence that typically involves altering structured priorities (diSessa 1993) of relevant knowledge elements. Conceptual change, in this perspective, involves altering the structured priorities of knowledge elements - i.e., altering the likelihood that particular pieces of knowledge will be activated upon recognition of specific contextual cues. In this view, misconceptions cannot easily be 'removed and replaced' because they are productive pieces of knowledge that are valid in certain contexts. In order to develop coherent understanding from pieces of knowledge, these naïve conceptions must be reorganized and refined into more stable, expert-like knowledge structures (Clark 2006; diSessa 1993; diSessa et al. 2004). As diSessa (1983) pointed out, physics learners tend to make errors because they overgeneralize - i.e., they use certain p-prims to make sense of situations in a manner that leads to erroneous explanations of the underlying physical mechanisms. Through instruction and experience, the student learns to activate different, more productive p-prims when presented with the same situation. Over time, similar contexts cue the more productive p-prims, thus modifying the structured priority of p-prim activation and building a more expert-like knowledge structure (diSessa et al. 2004).

From a KiP perspective, digital games can provide a context to support students in refining their structured priorities around targeted disciplinary relationships. An example of a disciplinary relationship that a digital game could target would be the shift from an emphasis on the Force as Mover p-prim to the Force as Deflector p-prim (diSessa 1988, 1993; White 1984). The Force as Mover p-prim assumes that a force causes motion in the direction of the force, ignoring the effect of previous motion (diSessa 1988; 1993). diSessa (1983) explains that the most commonplace situation involving forces, pushing on objects from rest, becomes abstracted as the highest priority p-prim that one will use to predict motion in general circumstances' (diSessa 1983, p 30). diSessa also pointed out that even experts likely use this p-prim to explain situations such as this one. However, the difference between novice and expert use of this p-prim is that the expert priority system 'knows' much better when and when not to use that intuition (diSessa 1993, $\mathrm{p}$ 130). diSessa argued that development of a more expert understanding involves raising the priority of a competing p-prim, Force as Deflector. Similar restructuring of priorities is critical to understanding other Newtonian relationships between force, mass, and acceleration as well as the interaction of multiple objects (diSessa 1993; diSessa et al. 2004). Designing games around conceptual integration focuses on supporting students in restructuring these cueing priorities through experiences in the games. Such designs can be informed by microgenetic analyses of student thinking and learning from a KiP perspective (and can leverage a long history of developing simulations and microworlds toward these goals).

\section{The science as practice perspective: games as model-based learning}

The Science as Practice (or SaP) perspective (Pickering 1995; Lehrer and Schauble 2006a; Duschl et al. 2007) argues that the development of scientific concepts is deeply intertwined with the development of epistemic and representational practices (e.g., modeling). Modeling is generally recognized as $a$, if not the, core disciplinary practice in the development of scientific expertise (Giere 1988; Nersessian 2002; Lehrer and Schauble 2006b; Duschl et al. 2007). Modeling involves the iterative generation and refinement of inscriptions (Latour 1990; Lehrer et al. 2000). As Latour (1990) originally explained, inscriptions refer to marks on a medium, such as paper or an electronic screen, and scientists design inscriptions in order to represent salient aspect(s) of the phenomenon under inquiry. Philosophers of science have pointed out that the central activity of science is the generation and testing of these models (Giere 1988; Hesse, 1974).

Models in science are explanatory in nature - i.e., they provide mechanistic explanations of natural phenomena (Giere 1988; Nersessian 2008; Lehrer and Schauble 2002). We can define models as fictive representations of real things (e.g., planes, cars, or buildings) or of systems (e.g., atomic structure, weather patterns, traffic flow, ecosystems, or social systems) that are simpler than the real objects and systems they represent but 
preferentially highlight certain properties of the referent (Rapp and Sengupta 2012). Giere argues that scientific explanations are constructed with models that have been developed in the sciences: 'Little can be learned ... about science that could not be learned more directly by examining the nature of scientific models and how they are developed' (Giere 1988; p. 105).

Specifically in the domain of kinematics, which is the focal domain of our own research, Hestenes (1993) argued that 'modeling is the name of the game' (p. 1) and that 'modeling is the main activity of scientists' (p. 6). Furthermore, Hestenes (1992) explains that modeling in kinematics involves three practices: development of a model; exploring the ramifications of the model in order to investigate the complex interactions in the phenomena; and deployment of the model in order to iteratively match it to empirical phenomena and data. According to Hestenes, the games of scientific explanation and prediction are key facets of model deployment. 'A physical phenomenon can be explained by a theory only to the extent that it can be modeled within the theory. Thus, the model is the explanation! [...] In a prediction game, the model is usually more explicit, because it is needed to generate some trend in simulated data' (Hestenes 1992, p 736-737).

Science and math education researchers have shown that engaging in modeling and progressively refining one's representation of some aspect of the world (e.g., a model or an inscription) can contribute to a deeper understanding of mathematical and scientific knowledge and practices (Gravemeijer et al. 2000; Hall and Stevens 1995; Lehrer and Pritchard 2002; Lehrer and Schauble, 2002). Enyedy (2005) refers to this process as 'progressive symbolization'. Lehrer (2009) makes clear that, while students need to see and handle models of abstract concepts in order for meaningful conceptual change to happen, the nature of the model is key. Not only do representations capture essential dynamic features of the relationships they describe but they also edit those relationships, foregrounding some elements and obscuring or omitting others (Lehrer and Schauble 2002; Lynch 1990). This 'editing' can potentially result in desirable as well as undesirable consequences for learners in terms of the structures and relationships recognized and interpreted by the learners as salient.

Can digital games as a medium support productive modeling and progressive symbolization? Gee (2008) discusses two characteristics supporting an affirmative answer: (1) games can enable the player to take on an empathetic perspective and (2) games can engage students using models and modeling as supports toward mastering relevant content knowledge. These two characteristics represent important meeting points between games and science education. The first characteristic, empathetic perspective, refers to a point of view a person can take in which they 'think like' the objects that they are investigating. Gee (2008) pointed out that taking the perspective of objects being investigated often plays a crucial role in the development of scientific understanding. A well-known example of this perspective in action can be found in Fox-Keller's biography of Barbara McClintock, in which the Nobel-prize winner describes how she achieved a major breakthrough in her research by learning to think like the DNA molecules that she was investigating (Fox-Keller 1983). Wilensky and Reisman (2006) and Dickes and Sengupta (2013) demonstrated that the ability to take on the perspective of thinking like the individual elements in a complex system can enable novice science learners to develop a deep understanding of the system. Gee argues that welldesigned games accomplish exactly that

Video games, under the right circumstances, may well be able to encourage (and actually help players to enact) an 'attitude' or 'stance' similar to the one taken by scientists studying complex systems. This stance involves a sort of 'embodied empathy for a complex system' wherein a person seeks to enter imaginatively into a system, all the while seeing and thinking of it as a system, rather than as a group of local or random events. (Gee 2008, p 32).

Gee's second point pertains to the use of models and modeling as key interactive elements in games. Gee points out that 'models and modeling allow specific aspects of experience to be interrogated and used for problem solving in ways that lead from concreteness to abstraction' (Gee 2008, pp 30). However, as Gee (2008) points out, the fact that a game deploys models does not automatically imply that the game play will necessitate modeling or the use of models. In some cases, for example, models within games are used primarily to drive and manage the context within which players themselves act (e.g., World of Warcraft). Focusing on disciplinary integration is intended to move students beyond acting within models to instead engage students in modeling as a means of predicting, controlling, and reflecting on actions and events in the game environment.

\section{Early focus on conceptual integration}

We began our own work on digital games and science learning with the goal of helping students integrate intuitive and formal understandings of Newtonian dynamics. We initially conceptualized the design as integrating (1) disciplinary representations and formal relationships of Newtonian dynamics with (2) popular recreational game mechanics from games such as Mario Galaxy and Switchball that include marble motion. The first game we 
developed and studied, SURGE Classic, takes places in an outer-space environment (Figure 1). Students use the arrow keys to navigate a rocket-powered spaceship (piloted by the 'Surge' avatar) around barriers and through corridors in search of non-player characters in need of rescue. Overlaid on the screen are different read-outs of information for players, including their ship's current speed, velocity vectors, the number of impulses used, the number of collisions with the walls, and elapsed time on a given game level. Players must minimize collisions, level completion time, and number of impulses used in order to earn a high score. Some levels also include a motion map region, where students must maintain a constant velocity, increase their speed, or decrease their speed in order to continue onward.

SURGE Classic (and subsequent SURGE designs) are 'conceptually integrated games' for learning (Clark and
Martinez-Garza 2012), as opposed to merely 'conceptually embedded games'. This means that the science to be learned is integrated directly into the mechanics as the central focus of navigating or manipulating the game world, rather than being embedded as an activity to be visited at some location in the game environment (i.e., isolated from the process of navigating or manipulating the overall game word and confined to challenges experienced at particular moments in time and/or special locations in the game world). The latter structure is typical of many virtual worlds designed for science learning. Note that both conceptually integrated and conceptually embedded games can represent intrinsic, as opposed to extrinsic, game design (c.f., Habgood and Ainsworth 2011). As an example, the intrinsic version of Habgood and Ainsworth's Zombie Division game is conceptually embedded rather than conceptually integrated

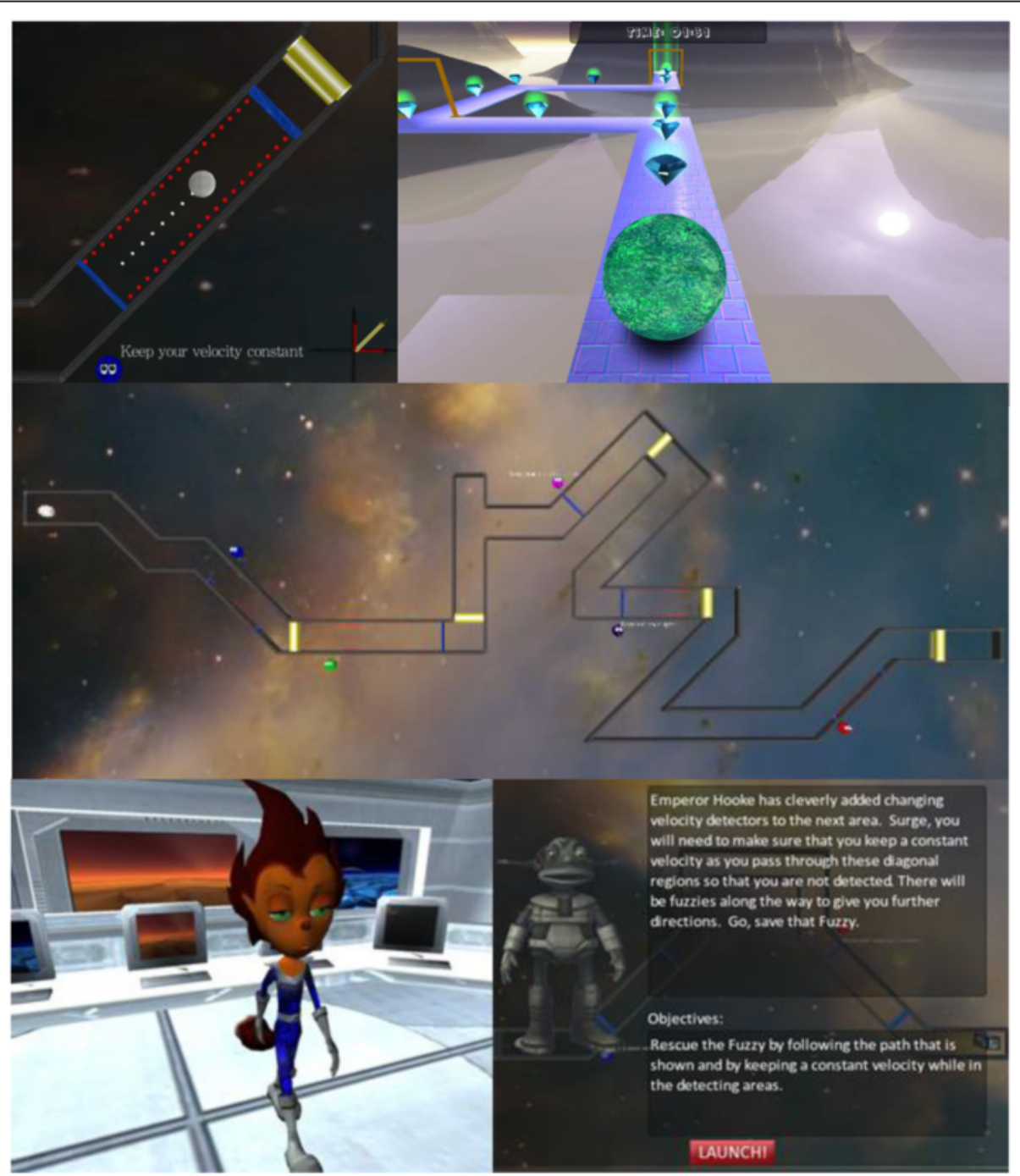

Figure 1 Screenshots from an impulse level in SURGE. Students must guide Surge in her spherical spaceship through maze-like prisons to rescue Fuzzies. 
in the sense that, while the math solution activity is intrinsic in the game, it is embedded rather than integrated into the fabric of navigating the world.

Conceptual integration required a two-pronged design approach: (1) designing levels around popular game-play mechanics from appropriate game genres, while also (2) designing challenges that directly explore, rather than merely contain, targeted physics relationships. Along the first dimension, SURGE Classic adopted core ideas from recreational game design conventions including (a) supporting engagement and approachable entry (Koster 2004; Squire 2011), (b) situating the player with a principled stance and perspective (McGonigal 2011), (c) providing context and identification for the player with a role and narrative (Gee 2007a; Pelletier 2008), (d) monitoring and providing actionable feedback for the player (Annetta et al. 2009; Garris et al. 2002; Munz et al. 2007), and (e) using pacing and gatekeeping to guide the player through cycles of performance (Squire 2006).

Along the second dimension, SURGE Classic game levels were designed to require students to apply and coordinate many principles related to Newtonian dynamics to complete levels (e.g., impulse, inertia, vector addition, elasticity of collisions, gravity, velocity, acceleration, free-fall, mass, force, and projectile motion). Physics ideas and terminology were built into pre-level and post-level story and feedback screens and within the levels of the game itself. The levels were designed so that physics concepts built upon one another and gradually introduced the student to new ideas and ways of interacting with the game world. For example, a student would first navigate through an orthogonal maze (which students could solve by using impulses to bring Surge to a stop in one direction before initiating movement in the perpendicular direction) before having to pass through mazes with diagonal corridors (requiring students to achieve states of motion that have non-zero velocities in both coordinate dimensions at once). Each level highlighted one or two topics, and levels allowed students to connect the concepts together and to see the relationships among the topics.

Throughout the levels, we attempted to minimize frustration for novice players. We learned, however, that protecting students from failure must not outweigh requiring mastery for progress. Clark et al. (2011) elaborate on these SURGE Classic design approaches in greater detail, but the descriptions here are sufficient to ground our discussion of the implications of lessons learned from SURGE Classic for subsequent phases of our game design, as well as for games for science learning more generally.

Students playing versions of SURGE Classic demonstrated high engagement and modest but statistically significant learning gains on items based on the Force
Concept Inventory (FCI), which is a widely known benchmark assessment for conceptual understanding of Newtonian dynamics at the undergraduate level (Hestenes and Halloun 1995; Hestenes et al. 1992). A study with seventh and eighth grade students in Taiwan and the United States (Clark et al. 2011) showed significant pre-post gains with modest effect sizes. Furthermore, students of both genders in both counties liked playing the game. Similar findings were obtained in four further studies conducted with US undergraduate physics students (D'Angelo et al. 2010), US Title I sixth grade students, and US undergraduate educational psychology students (Slack et al. 2010, 2011). Students' gains and increased mastery, however, focused on intuitive understanding (which is what the FCI largely measures) rather than competence with formal terminology or notations. Essentially, players could more accurately predict the results of various actions, impulses, and interactions (which improves performance in the game and on FCI questions), but players were not being supported in explicitly articulating the intuitive relationships in terms of formal disciplinary representations and concepts.

\section{Toward modeling and science as practice: prediction, reflection, and articulation}

Building on the findings from SURGE Classic, we began to explore ways to incentivize prediction and explanation to enhance conceptual integration in our next two game platforms, SURGE Next and Fuzzy Chronicles. A growing body of research and scholarship on games and cognition emphasizes informal cycles of prediction, explanation, and refinement at the core of game-play processes (Games-toTeach Team 2003; Salen and Zimmerman 2004, Wright 2006). Research in science education has demonstrated that prediction and explanation can scaffold students in reflecting more consciously and deliberately about the underlying physics models (e.g., Scott et al. 1992). Prediction and explanation can also promote metacognition, learning, and reflection (e.g., Champagne et al. 1982) as well as conceptual change (Tao and Gunstone, 1999; Kearney 2004). We have found, however, that design principles from research on learning often require adaptation for the digital game medium in a manner that is synergistic with, rather than disruptive to, the conventions and affordances of the medium.

\section{Integrating prediction into game play}

As in SURGE Classic, players in SURGE Next and Fuzzy Chronicles navigate their avatar through the play area to rescue game characters and deliver them to safe locations while avoiding obstacles and enemies. We changed the mechanisms of navigation, however, to emphasize prediction/anticipation rather than reaction. SURGE Classic employed a real-time reactive navigation structure where 
(a) pressing an 'arrow key' resulted in immediate application of an impulse or constant thrust in the direction of the arrow key and (b) motion in a desired direction often required continual split-second adjustments. In contrast, SURGE Next and Fuzzy Chronicles explored approaches to incentivize prediction by (a) creating challenges that required players to make fewer but higher impact decisions and (b) requiring players to spatially place all of these commands in advance. First, we reduced the total number of commands players initiate in a given level (thereby increasing the salience and impact of each individual command) to encourage players to think more carefully about the outcomes and implications of each action. Additionally, we required players to pre-place their solution rather than enact it in real time. Thus, in order to move along a particular path, the learner creates a predictive model of the trajectory by placing impulses along the target path (Figure 2). The learner then deploys his or her model by 'launching' the level to 'run' the plan. Players watch their plans unfold and then revise them accordingly.

\section{Microgenetic outcomes and implications}

In order to investigate the students' sense-of-mechanism (diSessa 1993) of the underlying physics, we conducted a microgenetic study in a ninth grade classroom in a Mid-Southern US public school with an early version of SURGE Next (Krinks et al. 2013; Sengupta, Krinks, and Clark: Conceptual Change in Physics through Use of Digital Games, revised and resubmitted). The data took the form of semi-structured clinical interviews that were conducted with each student upon successful completion of each level. We observed one particular conceptual
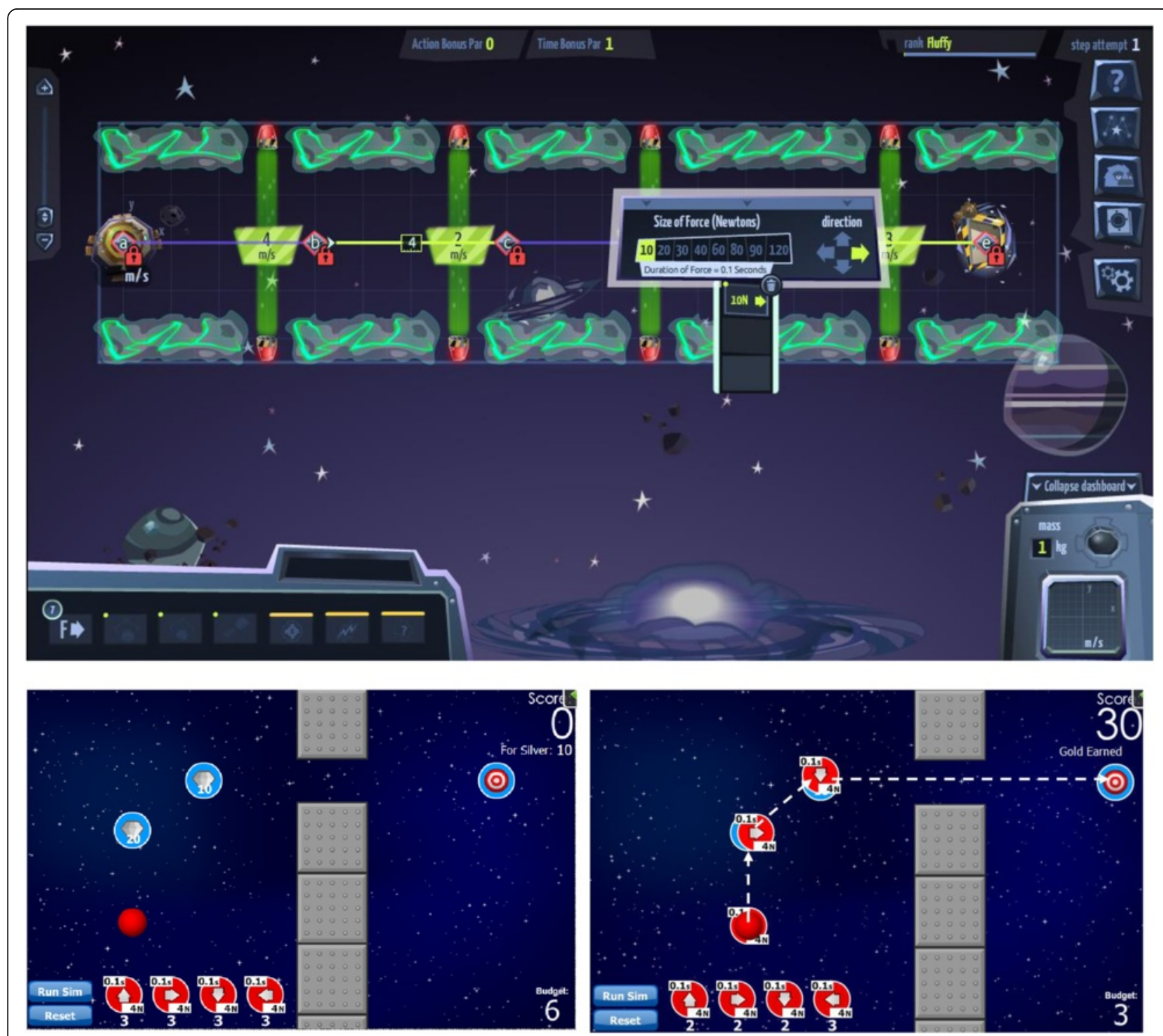

Figure 2 Simple example Fuzzy Chronicles level (top) and simple SURGE Next level and solution (bottom). 
resource that was deeply entrenched in the minds of students and manifested itself in many ways throughout all of the students' game play.

As mentioned in our overview of KiP, shifting to correctly prioritizing the Force as Deflector p-prim is key to understanding formal Newtonian relationships underlying Newton's first law. diSessa explained the schematization of the Force as Deflector p-prim as follows: 'A force (e.g., shove) may act in concert with prior motion (momentum) to produce a compromise result, directionally between the two' (diSessa 1993, pp 218). From the perspective of canonical physics, while Force as Mover neglects the role of the momentum of an object, Force as Deflector takes that into consideration. Therefore, the latter enables us to correctly predict and explain situations in which application of two impulses on an object along two different directions may result in motion of the object along a 'compromise' direction.

In our study, the Force as Mover p-prim was initially the preferred resource among all students. Through the conceptually integrated nature of game play, however, students realized that their intuitive physics ideas did not hold true in all situations. If they wanted to successfully solve the levels, students were compelled to consider properties of forces that they may never have consciously considered. Each level in the game acted as a sandbox in which students could easily model and test their intuitive ideas about the relationships between force and motion and receive immediate feedback as to the appropriateness of those ideas. In this learning environment, we saw students discover that certain ideas were productive in certain situations, thus activating resources in a new situation and reorganizing knowledge elements into a more cohesive framework. These newly cued ideas were the intuitive foundations of Newton's first law, such as the notion that an object will continue to move in its original direction until another force acts on it.

During the initial levels, we observed that students tenaciously used the Force as Mover p-prim, even after it had proven unsuccessful in producing the target outcomes in similar situations in the immediately preceding levels. To be clear, Force as Mover is not a 'wrong' resource that students should never use. On the contrary, it can correctly explain commonplace situations in everyday life, as pointed out earlier. But it does not always work well as an explanatory resource in more complex situations that involve the combinations of forces along different directions. Levels in SURGE Next deliberately expose students to situations where Force as Mover is not productive, enabling students to iteratively revise and refine their reasoning through re-designing, re-deploying, and re-assessing their solutions. The nature of the solution process itself - i.e., the placement of impulses along the predicted path of Surge - made explicit students' underlying assumptions, and the ability to verify, revise, and refine their solutions offered them opportunities to reflect and consider other conceptual resources. Gradually, new resources such as Force as Deflector emerged and increased in cueing priority, but only after repeated attempts and failures to make the primary resource of Force as Mover work. Similar to Gee's argument, we found that modeling Surge's motion through the placement of impulses along their predicted paths allowed specific aspects of the players' interpreted experience to be interrogated and used for problem solving in ways that led from concreteness to abstraction.

Eventually, these resources were cued with a higher priority than Force as Mover, as students became more adept at recognizing scenarios in which these resources were appropriate. Students' intuitive thinking in SURGE Next evolved, showing productive growth toward expertlike reasoning during game play. Thus this work highlights conceptual change as a process of refining cueing priorities and conditions rather than conceptual replacement. Furthermore, this work demonstrates the importance of students' conscious awareness of the outcomes from individual navigation decisions for the conceptual change process.

\section{Teacher thinking with SURGE: a case study}

Around the same time as the microgenetic study, we conducted a case study of a teacher and his students using an early version of Fuzzy Chronicles (Van Eaton et al. 2013). The study explored how the teacher and students interacted with the representations in a version of Fuzzy Chronicles that included a timeline structure for placing navigation commands. The goal was to understand how the representations affected the teacher's and students' epistemologies of force. We had hoped that the teacher's and students' understanding of force and motion would become more nuanced and that they would connect their intuitive understanding of physics with formal, canonical understanding of Newton's laws. The study highlighted improvements in the teacher's and students' ability to make phenomenological predictions about what would happen next given a configuration of impulses and their ability to manipulate the representations in the game to successfully complete levels, but it also highlighted how the teacher and students ascribed subjective properties to the representations of forces in the game to reconcile phenomena and relationships in the game with their own intuitive understanding of Newton's laws.

The teacher's and students' thinking paralleled Aristotelian notions of force being 'used up' or 'spread out' over distance. Thus distance was foregrounded in student or teacher's intuitive thinking about force (i.e., force 
as a means to travel a set distance, not accelerate the object to travel at a specific velocity for a set time). The teacher and students reinterpreted structural features of the representations in the game to support these understandings and subsequently used these structural features to reconcile their intuitive understandings when discussing the game in terms of formal Newtonian definitions of relationships. In these discussions, the teacher and student were able to 'bend' formal statements of Newton's three laws in terms of the representations in the game to warrant their intuitive thinking in nonnormative ways, such as interpreting forces applied against the direction of motion in terms of the wording Newton's third law.

We interpreted the case study findings as interactions between the representational nature of the game and the abstract concepts the game was intended to demonstrate (Van Eaton et al. 2013). As discussed earlier, Lehrer (2009) makes clear that students need to see and handle models of abstract concepts in order for meaningful conceptual change to happen; but the nature of the model is also key. Not only do representations capture essential dynamic features of the relationships they describe, but they also edit those relationships, foregrounding some elements and obscuring or omitting others (Lehrer and Schauble 2002; Lynch 1990), potentially both in desirable and undesirable ways. The representations we had designed in Fuzzy Chronicles scaffolded and refined the teacher and students' intuitive understanding of the relationships but are not sufficient to support a fundamental reinterpretation that the teacher and student could reconcile with normative interpretations of the relationships. Instead, the teacher and student reinterpreted structural features of the representation in ways to support their pre-existing non-normative interpretations. This case study thus explored the affordances of the representations and model they inscribed as well as the challenges of focusing teachers and students on the salient aspects of representations to support conceptual change. In other words, the case study highlights the intertwined relationship between focusing on representations, and the conceptual resources that are at play when teachers and students interact with the game.

\section{Experimental outcomes and implications}

In addition to the implications outlined above, our pilot studies with early versions of SURGE Next suggested that our approach to emphasizing prediction/anticipation rather than reaction was an improvement over our efforts in SURGE Classic. At the same time, however, these pilot studies showed how situation-dependent interaction design can be. Our intention was to incentivize predictive rather than reactive play. One key aspect of our approach involved requiring students to 'pre-place' the motion plan of their attempted solution, laying out impulses in space as opposed to enacting these impulses in real time. The other key aspect of our approach involved reducing the total number of navigational decisions/actions involved within individual game levels to increase the salience and consequence of each decision.

To explore the affordances of the first aspect of our design plan, we created two versions of SURGE Next, one requiring the player to pre-place navigational controls and the other requiring her to enact Surge's motion in real time. In line with the second aspect of our design plan, game levels in both the real-time and the preplaced versions focused on applying a small number of impulses of the rather than the large number of rapid actions required by SURGE Classic. To our surprise, quantitative pilot studies comparing the two versions of SURGE Next showed that both approaches had advantages (Clark et al. 2013), suggesting that the critical benefit for both derives from focusing game-play on fewer more consequential decisions so that players anticipate and think carefully about decisions and actions (i.e., engage in prediction). The pre-placed approach encouraged extended prediction of a full level solution but allowed continual revision of the plan, while the realtime version allowed for planning but also incentivized careful attention and provided immediate feedback as an outcome for each choice.

Thus, both approaches supported prediction/anticipation in different ways. Interestingly, attentional cognitive covariates (Rueda et al. 2004) for individual students correlated with learning gains differentially across approaches in these studies (Clark et al. 2013). This has motivated an ongoing line of research in our project to better understand how cognitive and motivational factors of players interact with different approaches to structuring navigation to support prediction, reflection, and learning (e.g., Killingsworth, Adams, and Clark: Cognitive and Motivational Influences on Learning and Play in a Physics Game, submitted). We hope through this research to develop approaches to adapt navigation structures for students based on their profiles and game-play logs. In terms of the current manuscript, however, the findings from this research on SURGE Next underscore (1) the importance of incentivizing prediction/anticipation and (2) the emphasis of learning gains on intuitive understanding.

Research on Fuzzy Chronicles has simultaneously compared (1) pre-placed approaches that engage students in placing actions spatially on the level map with (2) preplaced approaches where students pre-place actions in a timeline to emphasize the temporal relationships of motion rather than the positional relationships (Figure 3). As with the comparisons in SURGE Next, we are finding that different structures emphasize different affordances and cognitive demands by highlighting different relationships 


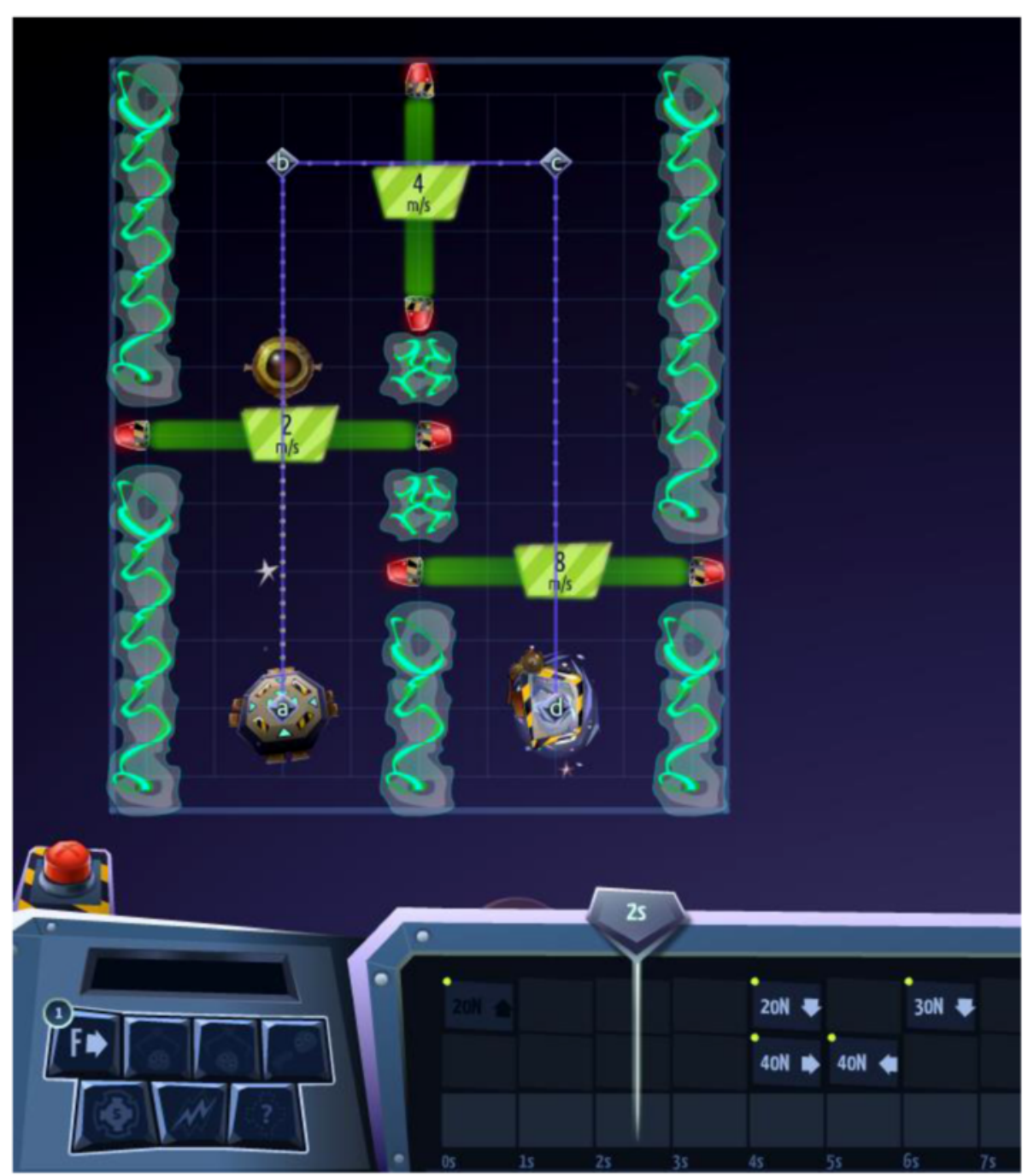

Figure 3 Simple Fuzzy Chronicles level with timeline placement of impulses to emphasize temporal relationships.

and aspects of the models structured by the students. The design challenges involve balancing these affordances and demands in a manner that allows students to focus on the core Newtonian relationships in a predictive, anticipatory, and conscious manner. The findings with Fuzzy Chronicles also parallel the findings from SURGE Next in their emphasis on the value of puzzle-like challenges with smaller numbers of high-salience decisions to support conceptually integrated designs and learning goals.

\section{Self-explanation to scaffold articulation}

To complement our increased emphasis on prediction in Fuzzy Chronicles and SURGE Next, we also began researching explanation functionality to scaffold students' articulation of the relationships they are exploring. Few games provide coherent structures for externalizing and reflecting on game play; more often, such articulation and reflection generally occur outside the game, through discussion among players or participation in online forums (Gee 2007b; Squire 2005; Steinkuehler and Duncan 2008). We are now working to develop supports for this articulation and reflection by encouraging explanation in the dialog between the players and computer characters.

Research on self-explanation by $\mathrm{Chi}$ and others provides insight into the value of explanation for learning (e.g., Chi et al. 1989; Roy and Chi 2005; Chi and VanLehn 1991). Encouragingly, research by Bielaczyc et al. (1995) shows that instruction that stresses generating explanations improves performance even after the prompts that drive the explanations are discontinued. Mayer and Johnson (2010) conducted preliminary work in embedding selfexplanation in a game-like environment with encouraging results, including gains on transfer tasks. This emphasis on explanation is mirrored in research on science education. Work by White and Frederiksen (1998, 2000), for example, demonstrates the value of asking students to 
reflect on their learning during inquiry with physics simulations.

Interestingly, while many aspects of recreational game design are currently very sophisticated compared to most educational software, dialog in recreational games tends to involve relatively simple 'multiple-choice' dialog trees that are not difficult to create. Educational games can thus easily leverage this general approach and even improve upon it. After a player has completed a set of missions in Fuzzy Chronicles, a computer-controlled character in the game contacts the player and asks for help. The subsequent dialog prompts a player to select explanations to answer the computer character by explaining how the player's solution relates to more formal articulations of the relationships. Our goal is to present these invitations for dialog as puzzles that are engaging in their own right (Clark and Martinez-Garza 2012; Clark et al. 2012).

Adams and Clark (2014) conducted an experiment with an early prototype of the explanation functionality in Fuzzy Chronicles (Figure 4). The experiment compared three conditions. Students in the 'questions' version of the game chose explanations for key phenomena at specific moments in game play - before testing their solutions, after an incorrect solution, and upon successfully completing a stage (e.g., when asked why they needed to apply an impulse at the beginning of a level, students needed to choose 'according to Newton's first law the ship will not move unless an unbalanced force acts upon it'). Students in the 'tips' condition, by comparison, would receive a tip (i.e., be provided an explanation) at corresponding moments in play - when they started an attempt, after an unsuccessful attempt, and after completing the level. Finally, students in the control condition received feedback only about whether they succeeded on the level or not.
This first study taught us a great deal about the structure, timing, and placement of explanation functionality to manage cognitive load, engagement findings that have informed subsequent approaches. One take-home lesson involved managing the complexity of the dialogic interactions in the context of the game experience's overall complexity. In some prototype situations, adding the explanation questions increased the complexity of an already complex task, disrupting students' experience of flow (Csikszentmihalyi 1991; Kiili 2005). A challenge when designing games for learning is to balance flow with moments of conscious reflection for learning (Kiili and Lainema 2008; Mayer and Johnson 2010). Another take-home lesson involved the timing of the dialog episodes. In some instances, the explanation episodes worked against aspects of students' game play that served to 'segment' a level into smaller chunks to manage cognitive demands (c.f. Mayer 2009). Segmenting a level is useful metacognitive or 'epistemic action' (Kirsh and Maglio 1994) that facilitates cognition in challenging settings. We realized that future designs needed to balance complexity while still providing opportunities for students to articulate the relationships they are exploring.

These take-home lessons underscored the challenges in reengineering and applying the findings of research from one learning context to another, particularly to contexts as rich as digital games. We reengineered the dialog functionality based on these lessons (Figure 5) and are currently collecting data with the new design. The preliminary analyses suggest that these changes are substantial improvements and can scaffold students successfully in reflecting on their actions and improving learning gains (Clark et al. 2012). A repeated-measures ANOVA revealed that the full explanation condition

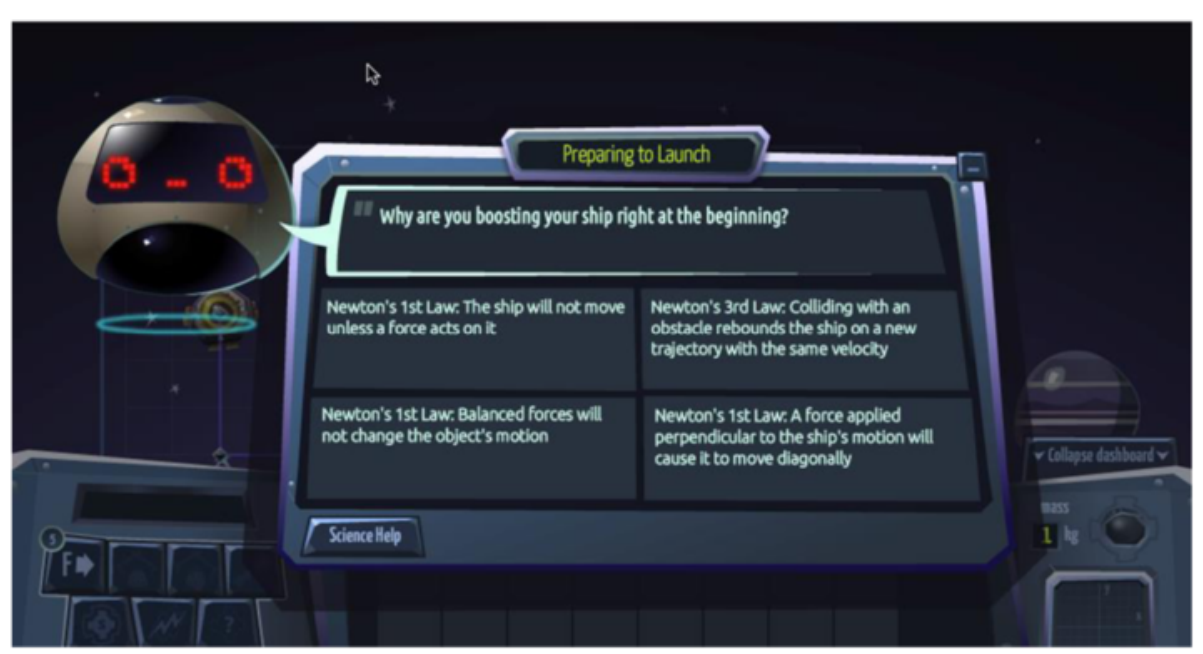

Figure 4 Early explanation functionality in Fuzzy Chronicles. 


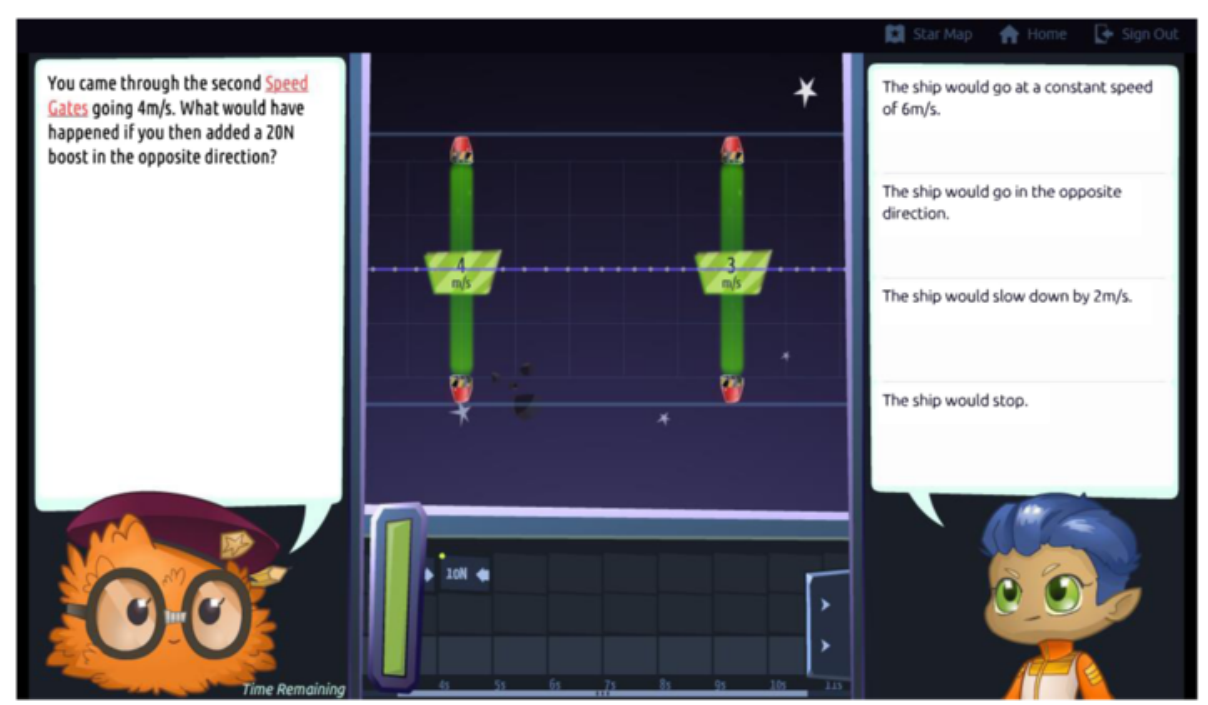

Figure 5 Revised explanation functionality in Fuzzy Chronicles.

outperformed the abbreviated explanation and tip conditions. Interestingly, although the full explanation condition required students to provide more answers and thus should have required more time, students in that condition actually completed slightly more levels in the game. This suggests that the full explanation conditions not only supported higher pre-post gains but also supported more efficient progress on the other parts of the game. We are currently conducting data-mining analyses of the data to explore these differences to better understand the mechanisms of learning supported through the full explanation condition.

Our results from this latest study have convinced us of the potential value of engaging students in the practices of explanation and articulation to enhance their understanding of the underlying relationships they are modeling in the game. We continue to refine and revise our design principles based on observations and data from the field clarifying synergistic approaches for integrating explanation and reflection into game play, as we will expand upon in a later section of this paper.

\section{Toward disciplinary integration: integrating graphing with prediction and explanation}

Through our work with integrating prediction and explanation into our designs, we have come to understand that conceptually integrated games need to create conditions for mathematizing situations and phenomena using domain-appropriate and task-appropriate symbolic representations. In this way, it is possible to engage students in modeling through game play. We developed this hypothesis in response to recurring patterns in findings across our studies. Nearly all students were able to develop productive intuitions about the underlying physical phenomena within the game as evident from the pre-post gains across studies, the microgenetic analysis (Krinks et al. 2013, Sengupta, Krinks, and Clark: Conceptual Change in Physics through Use of Digital Games, revised and resubmitted), the teacher case study (Van Eaton et al. 2013), and data mining analysis of log data from students' game play (Kinnebrew et al., in review). Students were generally not demonstrating strong abilities to connect that intuitive understanding across comparatively more formal, canonical representations of motion.

In order to address this issue, we set out to create additional opportunities in which students would engage in symbolizing salient aspects of motion phenomena and the underlying Newtonian dynamics. This symbolizing work was deeply integrated with the game experience so as to augment the effects of intuitive exploration and understandings achieved with prior designs. Our premise was that, as students progressed in our game, they should be able to design and utilize progressively more complex inscriptions of the kinematic phenomena they were exploring in the levels.

Designing these mathematical representations corresponds to model development, while utilizing them in order to accomplish a certain objective corresponds to model deployment, leading in turn to refinement of the model. Progressively increasing complexity involves generating and comparing multiple symbolic representations of the same phenomenon (e.g., dot traces and graphs of different types), as well as generating inscriptions of progressively more complex phenomena (e.g., situations involving constant speed, constant acceleration, and varying acceleration). We call this evolution of complexity progressive symbolization, appropriating Enyedy's (2005) use of the term. We envision progressive 
symbolization as a process of learning that can be supported through game play, by designing levels that progressively add symbolic depth and representational complexity to relevant game elements.

In our work, progressive symbolization within the game designs has proven valuable in several ways. First, the need for symbolization can be created within the narrative of the game; this enabled us to foster a deep engagement of the learners with the practice of modeling. Second, the symbolic representations generated by learners can be analogous to canonical representations that they will encounter in other contexts. This adds authenticity to learners' modeling work. Third, students have a meaningful experience of the relationship between work with representations on the one hand, and the development of knowledge and insights on the other hand - a recognition of the generative, constructive nature of working through representations to gain mastery of concepts and relationships. Fourth, students develop a deeper understanding of the underlying concepts and mathematical relationships. As argued earlier, this is also in tune with science and math education researchers' findings that engaging in modeling and progressively refining one's representation of some aspect of the world (e.g., a model or an inscription) can contribute to a deeper understanding of mathematical and scientific knowledge and practices (Gravemeijer et al. 2000; Hall and Stevens 1995; Lehrer and Pritchard 2002; Lehrer and Schauble, 2002).

To test these ideas, we extended SURGE Next to create SURGE NextG. SURGE NextG includes a graphing environment that enables real-time construction of mathematical representations based on periodic sampling of measures of Surge's motion (see Figure 6a,b,c). Students engage in modeling by generating, navigating between, interpreting, and reflecting upon both spatial records representing position in time (e.g., dot traces) and graphical representations of changes in features of motion over time (e.g., graphs).

Our goal with the design of Surge NextG was to further deepen the nature of students' modeling experiences by creating opportunities for students to engage in generating and reasoning about relations between multiple genres of inscriptions (e.g., dot traces and graphs of different features of motion, such as speed, displacement, and acceleration). This approach builds on a long history of having students match motion with graphs, control motion with graphs, or create graphs through motion with motion sensors. Some of the most detailed work to date in this area was conducted as part of the SimCalc projects, beginning with MathCars (Kaput 1994) and later MathWorlds (Rochelle and Kaput 1996; Hegedus and Roschelle 2013).

In SURGE NextG, students can generate multiple bar graphs, including an 'odometer' graph that shows total displacement as a function of time, graphs of signed $\mathrm{x}$ - and $\mathrm{y}$-component displacements, and a 'speedometer' graph that shows total velocity as a function of time, and graphs of signed $\mathrm{x}$ - and $\mathrm{y}$-component velocities. Each bar in the graph corresponds to a periodic measurement of distance traveled by Surge within successive time intervals - i.e., like the dot trace, each bar is the record of an instant, with the aggregate graphical representation emerging over time. Unlike with the dot trace, however, in the graphing environment, students can determine the frequency of measurements, either synchronizing them with the dot trace or making them more or less frequent. They can also alter several other features of the graph through provided controls. Finally, students can rewind and replay the completed level in order to examine temporal relationships between the graph and game motion more closely.

Incorporating graphing as a necessary interaction between the learner and the game (and thus shifting from conceptual integration toward disciplinary integration) has had additional implications and affordances for activity design. In particular, it has opened up opportunities for using graphical representations of plans, intentions, and predictions as mediators to connect virtual actions in the game with situations of physical motion in the real world. For example, in SURGE NextG, the students were asked to guide a ship through a nebula that was interfering with communication. Students watched a video of a ball first rolling down a ramp, then rolling up another ramp to reach its maximum height. Students were told this was a clue about how to guide the ship and asked to design a game level so that the ship's motion matched the motion of the rolling ball. Students were prompted to create and use graphs of the ship's motion when designing their level. Students were asked to draw a sketch of their solution and to explain how their design matched the video of the ball rolling on the ramp. Following the initial design, students were given 'updated information' from the captain in the form of a speed-time graph that the ship needed to generate and asked to redesign their solutions. Again, students were asked to sketch and to explain their designs.

We conducted a study with this version of SURGE $N e x t G$ with four sections of seventh grade students taught by the same teacher (Sengupta et al. 2014; Learning Physics through Representational Translation in Video Games, in preparation). The study led to interesting results that highlight the benefit of symbolization as a gaming action. Each section was assigned to one of two graphing conditions (grapher vs. no grapher) and one of two collaboration conditions (collaborative vs. non-collaborative). We found that students in the grapher sections showed significantly greater pre-post test gains on key Newtonian relationships. Furthermore, the grapher students who 

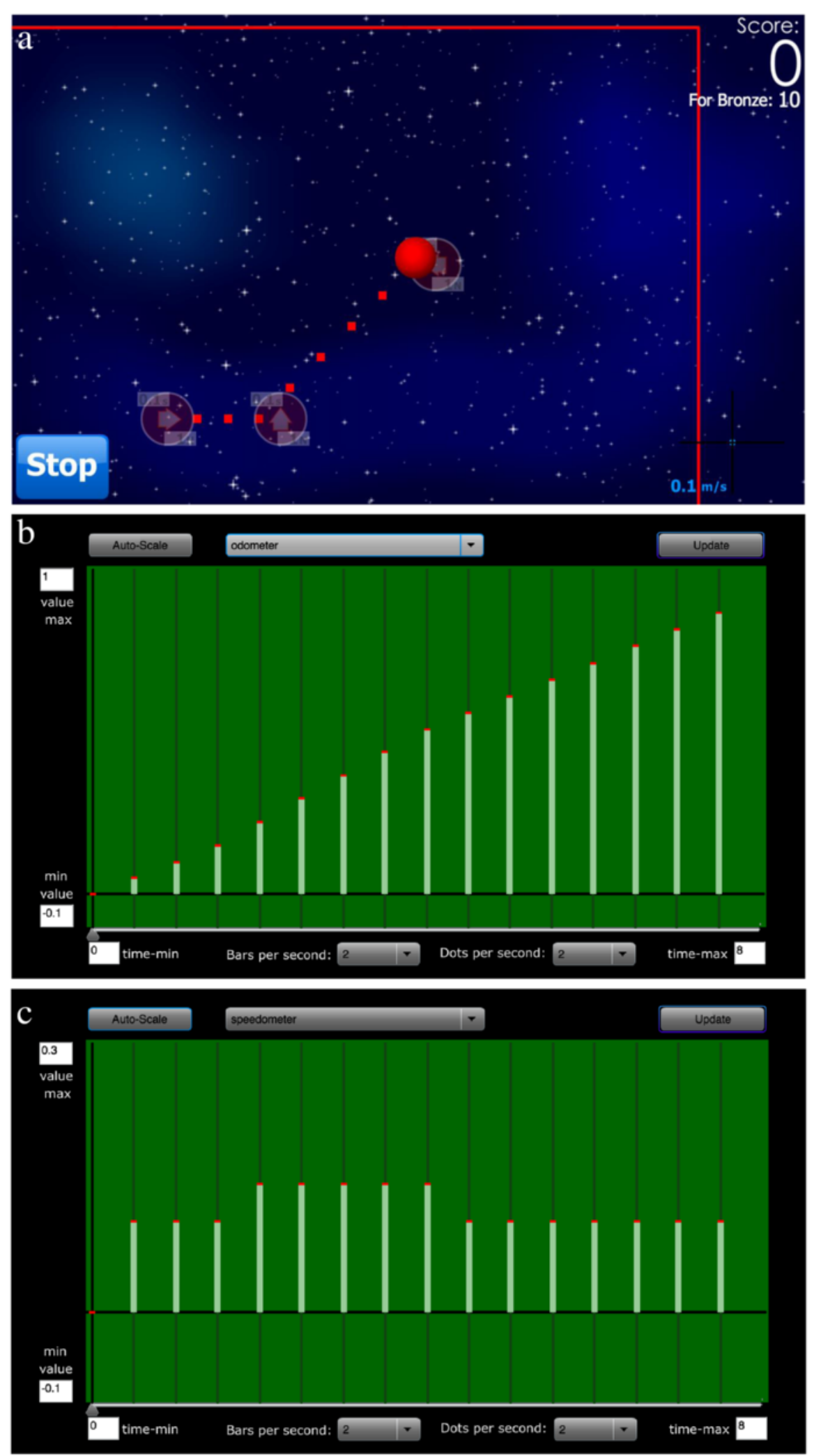

Figure 6 SURGE NextG. (a) A level depicting one-dimensional motion of Surge in SURGE NextG. (b) The displacement graph that accompanies the one-dimensional motion in SURGE NextG. (c) The corresponding velocity graph for that same motion in SURGE NextG. 
framed their plan in a variable-focused way (e.g., thinking about the meaning of the relationships in the graph) rather than experientially (e.g., in terms of the shape of the graph) showed the highest gains of all.

These findings highlight the importance of progressive symbolization for developing deeper understandings of Newtonian relationships within the game. As students progressed through the levels of SURGE NextG, the game became a modeling tool for iteratively developing formal representations (graphs of motion) and connecting these representations to situations of motion in the real world. In the earlier levels, the game play was designed to familiarize students with the core computational representation of motion - dot traces - by engaging them in designing predictive trajectories of SURGE. In later levels, students iteratively designed inscriptions in SURGE NextG in order to further mathematize the dot-trace representations and use these two representations to model an example of constant acceleration in the real world. In doing so, students developed explanations (models) of motion, made predictions, and deployed their models to verify and further improve on them - and in the process, developed a deep understanding of conceptual relationships in kinematics. This relationship between meaningful symbolization and the development of conceptual understanding is central to disciplinary integration.

\section{Deeper into disciplinary integration}

We are currently developing a new game, SURGE Symbolic (Clark et al. 2014b), that carries the principles of disciplinary integration further, deepening the symbolization experiences of players while still attempting to stay true to the roots and conventions of recreational games (Figure 7). Specifically, SURGE Symbolic centers game play in manipulating and navigating across formal representations while simultaneously engaging players in dialog with computer characters to articulate the relationships inscribed within and across those representations.

\section{Progressive symbolization}

SURGE Symbolic engages students in a process of progressive symbolization with an emphasis on iteratively developing representational fluency and conceptual understanding. Play begins in the informal space without access to the Cartesian graphs. Initially, players only have the dot-trace representation and vector arrows for force and velocity. Cartesian representations of position, velocity, and force are gradually introduced to provide the player with tools to exert greater levels of control

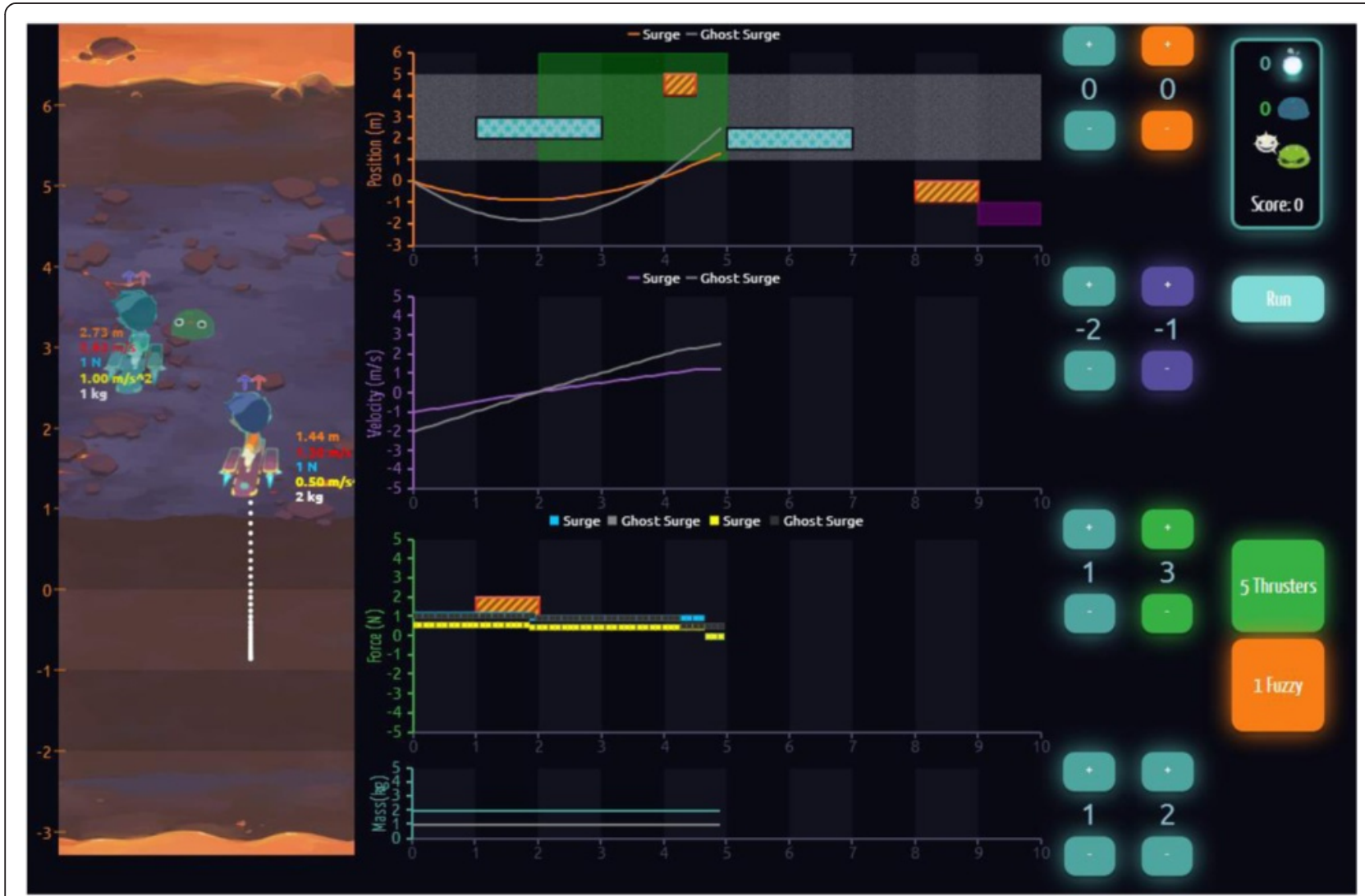

Figure 7 SURGE symbolic. 
and prediction over what unfolds in the world space. Moreover, increasing focus is placed on coordination across multiple graphs. Thus play shifts from the informal space deeper and deeper into representational space. Successful game play depends on developing the ability to place multiple relationships in conversation with one another - gleaning the information that each can provide and understanding each as an important but partial perspective on the activity of the game.

\section{Centering game play in the formal representations}

Whereas earlier versions of SURGE focused more on layering formal representations over informal representations, SURGE Symbolic inverts this order, layering informal representations over formal representations while organizing game play explicitly around navigating and coordinating across representations. This provides multiple advantages compared to previous versions of SURGE. While earlier versions supported reflection on the results of game play through formal representations as a means to support strategy refinement (particularly in SURGE NextG), the formal representations were not the medium through which players planned, implemented, and manipulated their game strategies. In SURGE Symbolic, the Cartesian graphs of position, velocity, acceleration, force, and mass are where the puzzles are presented to players, where players plan and implement their actions and where players reflect on the outcomes of their plans. The informal space (which displays the Surge avatar and its physical surroundings) augments the formal representations, but the Cartesian graphs are the hub of all planning, action, and reflection. The graphs are the way that the player communicates with the game (i.e., controls the game) and the way that the game communicates the goals, challenges, events, and outcomes to the player. This is the opposite relationship from earlier versions of SURGE, where the informal space provided the hub of activity, while the formal spaces augmented reflection. Thus the Cartesian graphs now provide the language of control and planning as well as the language of description and reflection.

The emphasis on the Cartesian graphs also provides another key advantage. The navigation structures in earlier versions of SURGE preferentially foregrounded position, time, or motion. SURGE Symbolic emphasizes the relationships among all three by utilizing the formal Cartesian graphs as the navigation inscription. This focus on the unifying relationships and links between Cartesian graphs provides a more powerful foundation for exploring key Newtonian ideas, which themselves focus on these relationships.

Our approach extends prior work in using graphs as communications and control structures with 'representational expressivity,' (Hegedus and Moreno-Armella 2009) in two critical ways. First, concerning graph-bound representations as communication structures, our approach uses the Cartesian graphs for strategizing and acting, in which critical information about upcoming challenges is communicated to the player through the 'language' of the Cartesian graphs themselves. The position graph, for example, can present information about the specific regions of the game-world that will be affected by dangerous electrical storms at given times, as well as about locations where rewards or allies will appear to rendezvous with Surge. The velocity and acceleration graphs likewise indicate constraints on game-play, such as maximum or minimum speeds and maximum accelerations that must be observed by Surge or non-player characters during specific time periods, each of which can be tied to narrative elements in game scenarios. Thus again, while positive and negative events also unfold in the informal representations of the game, the hub of all prediction, action, and reflection about these events is integrated into the Cartesian graphs. As a result of this design approach, the Cartesian space emerges as a set of scientific instruments for the player, in the sense of providing access to data about the game world that are not available through other means. At the same time, the Cartesian graphs also play the role of an instrument panel or mission planner, offering fine-grained control over the movement of the Surge spacecraft.

\section{Flexible dialog system to scaffold reflection and articulation}

A key aspect of disciplinary integration focuses on supporting reflection and articulation about the progressive symbolization in which the player is engaging. Toward this end, players in SURGE Symbolic will engage in conversational exchanges with non-player characters, with the goal of supporting players in externalizing and articulating elements of their strategies. Thus, progress in coordinating representations and seeing representations as being in 'conversation' with one another is fostered and documented through dialog exchanges between players and non-player characters.

Toward this end, we have developed an adaptive branching structure for the dialog. At the most basic level, this allows students' choices in one frame of the dialog to change what the student encounters in the next frame of the dialog (Figure 8). This is standard functionality in games. What is more novel is that we can also integrate game levels into frames of the dialog so that students can (or even sometimes need to) work on navigation challenges as part of their response in a dialog frame. Furthermore, the response options provided to a student in a dialog frame can adapt depending on the student's actions in the navigation portion of that dialog frame. 


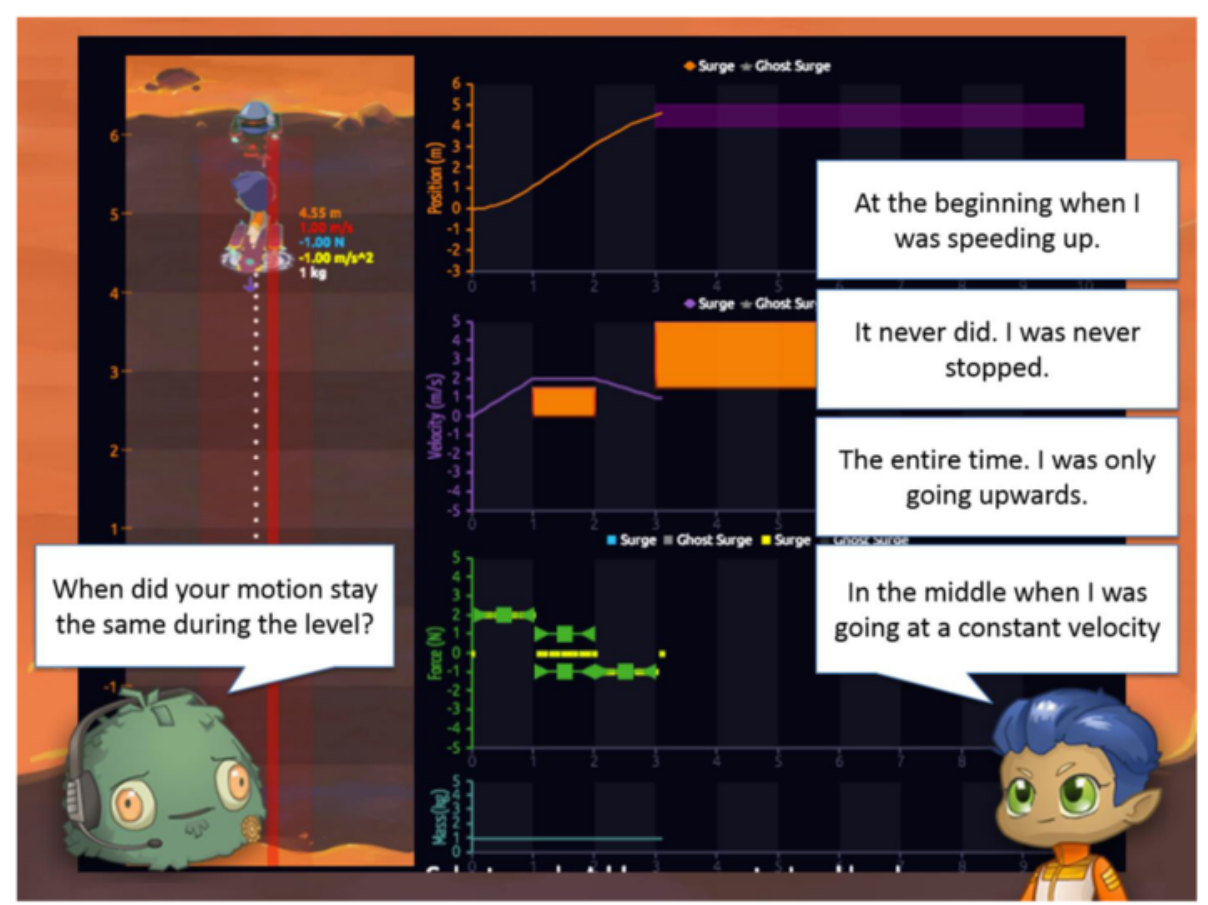

Figure 8 SURGE symbolic with integrated dialog.

Our goals for this functionality are threefold. First, we hope that providing more fluid and targeted responses to players' dialog choices will make the interaction feel more natural and engaging to players, thus increasing engagement and flow within the game.

Second, the new system allows us to develop adaptive scaffolding by adjusting students' experiences based on what they do in the game. If they struggle with a given challenge, they can be routed to additional experiences to make sense of that challenge. If they demonstrate mastery, their route can be expedited to more challenging encounters. If they make a dialog choice suggesting a misconception, the dialog can follow up with further dialog and exploration of those ideas. This functionality will allow us to build on our work with a hybrid p-prim/ facet framework of student ideas (diSessa 1993; Krinks et al. 2013, Minstrell 2001; Sengupta, Krinks, and Clark: Conceptual Change in Physics through Use of Digital Games, revised and resubmitted) that can manifest within game play as a means of tracking students' progress and adjusting their experiences.

Third, the dialog system will allow us to more seamlessly integrate experiences from outside of the game into the game to support progressive symbolization. Similar to SURGE NextG, players will use SURGE Symbolic to develop models of examples of motion in the real world, in later levels in game play. In addition to supporting progressive symbolization, this will support preparation for future learning (Bransford and Schwartz
1999; Schwartz and Arena 2013), as it will allow students to connect the physics represented in the game with scenarios involving motion in the real world.

\section{Generalizing disciplinary integration beyond Newtonian dynamics}

Can disciplinary integration can be abstracted and generalized beyond Newtonian dynamics to other core topics? We argue that it can. A powerful framework for generalizing disciplinary integration is provided by Allan Collins and colleagues in their analyses of 'epistemic forms' and 'epistemic games' (Collins 2011; Collins, personal communication, June, 30, 2014; Collins and Ferguson 1993; Morrison and Collins 1995). More specifically, Collins and colleagues argue that the professional work of scientists can be understood in terms of epistemic forms (model types that are the target structures guiding scientific inquiry) and epistemic games (modeling strategies that are the sets of rules and strategies for creating, manipulating, and refining those model types). Collins and colleagues are considering shifting their terminology from 'epistemic forms and epistemic games' to 'model types and modeling strategies' to increase clarity of intent to a broader audience (Collins, personal communication, June 30, 2014), but we will use the original terminology of 'epistemic forms' and 'epistemic games' here to disambiguate from our own use of the terms models and modeling. Collins and colleagues new terminology of 'model types and modeling strategies,' however, underscores the tight fit with 
the focus of disciplinarily integrated games on models and modeling.

Collins and Ferguson (1993) argue that the advantages of focusing on models and modeling strategies as curricular goals are that they allow educators to move beyond a primary emphasis on 'teaching facts, concepts, and problem solving methods, along with particular theories and models' (Collins and Ferguson, 1993; p. 26), to 'some of the more important forms and games' to guide students' inquiries (p. 26). While Collins and Ferguson did not write with the intention of informing the design of actual digital games (they used the term 'game' as a metaphor), disciplinarily integrated games can be framed as building on the ideas of Collins and colleagues by structuring digital game play around epistemic games of designing and manipulating formal disciplinary representations (epistemic forms). More specifically, if we frame the puzzles in disciplinarily integrated games as distilling epistemic forms (model types) and the epistemic games (modeling strategies) for navigating and manipulating those forms, we open disciplinary integration well beyond our focus on Newtonian dynamics to span across disciplines.

Disciplinarily integrated games are inscriptional systems, and it is particularly important to consider the pedagogical value of the different symbolic systems deployed within a game, as well as the practices (i.e., actions or sets of actions, and the associated goals) that the players need to use in order to use these systems. For disciplinary integration, the forms and practices within the game need to be tied to representations and practices that are needed in order to develop professional scientific expertise. We posit that disciplinarily integrated games should support a key professional practice that cuts across scientific domains modeling. This means that players' actions should involve the iterative development of inscriptions in the form of computational and mathematized representations of focal phenomenon in order to investigate key conceptual relationships in the domain, while also developing facility with the representations and inscriptions themselves. As mentioned earlier, this is at the heart of the 'Science as Practice' perspective: the history of science unambiguously shows that conceptual development in the sciences is deeply intertwined with the development of representational practices (Nersessian, 1998; Giere 1988; Pickering 1995; Lehrer and Schauble 2010).

Of note here is the use of multiple representational systems as tools for inscription. For example, in our work, dot traces and graphs are complementary representational systems, and when students used these systems together as part of their game play, they developed a deeper understanding of the target disciplinary ideas (i.e., concepts). Comparing how relevant aspects of important phenomenon can be represented using different inscriptional systems can support learners in developing a key characteristic of modeling expertise: communicativity (Lehrer, 2003; Lehrer and Schauble 2006b).

We therefore argue that disciplinarily integrated games engage students in playing epistemic games (leveraging modeling strategies) to manipulate and navigate challenges structured in epistemic forms (model types) as a means of (1) coming to understand those epistemic forms and epistemic games as important goals in their own right while (2) simultaneously coming to understand the disciplinary concepts driving the game. It is important to understand that this emphasis of disciplinary integration necessitates that we recognize that conceptual development and the development of representational practices (e.g., modeling) are deeply intertwined. This dual focus parallels the distinction in research on argumentation in the science classroom between 'learning to argue' and 'arguing to learn'. We assert that a joint focus on both is essential, because of their deeply intertwined nature. We therefore propose that disciplinary integration must target conceptual learning as well disciplinary learning in terms of the representational practices of the discipline.

One might then ask: how is designing for disciplinary integration different than designing a 3D virtual inquiry world (e.g., Quest Atlantis, River City, or Crystal Island)? We would argue that a key distinction in design involves the nature and breadth of focus. Virtual inquiry worlds generally engage students in the practices and Discourses (Gee 1990) of a discipline at the level of inquiry writ large. Much of the pedagogical power and engagement of 3D virtual inquiry worlds tends to focus heavily on their impressive affordances for role-playing, narrative, and identity-building (cf. Gee 2007a). While 3D virtual inquiry worlds are compelling and powerful, their scope and structure do involve tradeoffs in terms of the individual tasks or puzzles themselves, which are often themselves relatively mundane individually (e.g., click on a character to be told a piece of evidence, click on a location to get a reading on oxygen levels, or click on a location to bring up another mini-game to collect evidence).

Disciplinarily integrated games do not attempt the depth of immersion, identity-building, and role-playing of virtual inquiry worlds (and do not dispute their importance or value), but disciplinarily integrated games do afford potentially greater sophistication and refinement of the game-play surrounding the targeted epistemic forms and epistemic games by virtue of their tighter focus. Essentially, whereas 3D virtual inquiry worlds tend to cast students as scientists investigating a 'mystery' at the level of overarching inquiry, disciplinarily integrated games are more focused on specific epistemic games of manipulating specific epistemic forms. This focus allows for progressively deepening the puzzle at the heart of the game and all elements of the game, to emphasize the puzzle. 
Disciplinary integration thus focuses foremost on aligning game play with modeling around a specific central set of disciplinary inscriptions rather than engaging in 'inquiry' more broadly. Disciplinary integration is fundamentally generative in that students develop, refine, and deploy models as actions that constitute game play. As students progress through curricular sequences, the game provides a sandbox for modeling phenomena in the real world. Thus, disciplinarily integrated games emphasize STEM practices, but in very focused ways, with any given disciplinarily integrated game focusing on a subset of epistemic forms, or even a single epistemic form, rather than on broader practices (e.g., 'inquiry' writ large).

In terms of examples beyond Newtonian dynamics, we would argue that DragonBox meets our criteria for disciplinary integration (http://dragonboxapp.com). DragonBox engages players in manipulating algebraic equations through the actual syntax, structures, and procedures of formal algebra. Players isolate boxes to grow their dragons. Players can transform and move and eliminate boxes in various ways. Everything gets introduced in a cartoony, pictorial, non-mathematical way, but gradually, the pictorial representations are pared back until players are moving through screens of more standard algebraic syntax. Another example is provided by FoldIt, which adopts molecular schematics as the target epistemic form and engages players in structuring proteins to fold into specific spatial configurations (http://fold.it). The puzzles and mechanics in these games are highly focused (e.g., FoldIt's sequencing of molecules that will fold into specific shapes), providing clean, challenging, and engaging puzzles.

SURGE Symbolic, DragonBox, and FoldIt are but three possible examples for structuring disciplinary integration around epistemic forms and epistemic games. Framing disciplinary integration in terms of epistemic forms and games opens a vast trove of epistemic forms and epistemic games that span across disciplines (in fact well beyond STEM into the social sciences). Disciplinary integration of digital games thus provides a generalizable genre that holds promise as a vehicle for engaging students in key epistemic forms and games that cross multiple disciplines and respond to calls for greater emphasis on problem solving, 21st century skills, and engaging students in the practices of disciplines to develop deeper understanding.

\section{Reflections and final thoughts}

At the highest level, designing games for learning involves integrating learning goals, game design goals, and technology affordances. As with any design activity, game design is fundamentally a creative activity that is bounded not only culturally by the definitions, trajectories, and conventions of the genre it represents but also bounded materially by the economic and technological realities of producing the work itself. Unlike more historically established forms of design (e.g., architecture), game design has not fully explored the limits of its conventional boundaries. Cultural theorists like Berger (2002) advance the position that digital games are a 'remediation' of earlier text forms, while game design experts stress the novel representational and interactive capacities of the medium (Crawford 1984).

A designer of educational games must attend not only to the conventional forms of the particular genre and subgenre of the game that is being created but also to whether or not the chosen forms are proper carriers of the concepts, systems, and relationships of the learning objectives. This is especially problematic in games for science learning because some of the most powerful ideas of science come in the form of systems, models, and relationships. It is not sufficient to design a compelling game that engages students. Games for science learning should also incorporate the models and structures that support the learning goals and optimize players' learning processes in light of those goals. In this regard, some game forms and concepts are likely more conducive to specific science learning processes and goals. Ultimately, the work of the designer of a game for science learning involves determining which specific conventions of game design support students' engagement with which specific science learning goals and processes.

This paper establishes learning theory as an essential guidepost for dealing with these design challenges and objectives. With KiP as an underlying theory, our early emphasis was on conceptual integration, as evident in the early versions of SURGE Classic and SURGE Next. Our design choices in these versions were driven by one key goal: to enable students to better articulate their intuitive understandings of the underlying physics. The iterative nature of game play provided students multiple opportunities of prediction and self-explanation, and in the process, supported students in productively reorganizing their understanding of the Newtonian relationships. In later versions of SURGE, we further emphasized practices central to modeling - both epistemic and representational - in order to support conceptual development. This was a result of our shift from KiP to SaP as the underlying theoretical framework. Game play now focuses on modeling through manipulation and navigation of formal disciplinary representations - a perspective that we have termed disciplinary integration.

\section{Competing interests}

The authors declare that they have no competing interests.

\section{Authors' contributions}

DC and PS are the equal lead authors and conceptualizers of the new thinking, designs, and writing in the manuscript. CB contributed to the authoring of the discussion about SURGE Symbolic and contributed by collaboratively implementing an early prototype of the SURGE Symbolic 
software and the graphing components of SURGE NextG. MM-G and SK contributed to the authoring of other specific sections. All authors read, edited, and approved the final manuscript.

\section{Authors' information}

The research reported here was supported by the National Science Foundation through grants 1019170 and 1119290 and the Institute of Education Sciences, U.S. Department of Education, through grant R305A110782. The opinions expressed are those of the authors and do not represent views of the Institute, the U.S. Department of Education, or the National Science Foundation

\section{Author details}

${ }^{1}$ College of Education, Vanderbilt University, Box 230, 230 Appleton Place, Nashville, Tennessee 37203-5721, USA. ${ }^{2}$ School of Education and Social Policy, Northwestern University, Annenberg Hall, 2120 Campus Drive, Evanston, Illinois 60208, USA.

Received: 4 September 2014 Accepted: 1 December 2014 Published online: 11 February 2015

\section{References}

Adams, DM, \& Clark, DB. (2014). Integrating self-explanation functionality into a complex game environment: Keeping gaming in motion. Computers and Education, 73, 149-159.

Amin, TG. (2009). Conceptual metaphor meets conceptual change. Human Development, 52(3), 165-197.

Annetta, LA, Minogue, J, Holmes, SY, \& Cheng, M. (2009). Investigating the impact of video games on high school students' engagement and learning about genetics. Computers \& Education, 53(1), 74-85.

Berger, AA. (2002). Video Games: A Popular Culture Phenomenon. New Brunswick, NJ: Transaction Publishers.

Bielaczyc, K, Pirolli, P, \& Brown, AL. (1995). Training in self-explanation and self-regulation strategies: Investigating the effects of knowledge acquisition activities on problem solving. Cognition and Instruction, 13(2), 221-252.

Bransford, JD, \& Schwartz, DL. (1999). Rethinking transfer: A simple proposal with multiple implications. Review of Research in Education, 24, 61-100.

Champagne, AB, Klopfer, LE, \& Gunstone, RF. (1982). Cognitive research and the design of science instruction. Educational Psychologist, 17(1), 31

Chi, MTH, \& VanLehn, KA. (1991). The content of physics self-explanations. Journal of the Learning Sciences, 1(1), 69-105.

Chi, MTH, Bassok, M, Lewis, MW, Reimann, P, \& Glaser, R. (1989). Self-explanations: How students study and use examples in learning to solve problems. Cognitive Science, 13(2), 145-182.

Clark, DB. (2006). Longitudinal conceptual change in students' understanding of thermal equilibrium: An examination of the process of conceptual restructuring. Cognition and Instruction, 24(4), 467-563.

Clark, DB, \& Martinez-Garza, M. (2012). Prediction and explanation as design mechanics in conceptually-integrated digital games to help players articulate the tacit understandings they build through gameplay. In C Steinkuhler, K Squire, \& S Barab (Eds.), Games, Learning, and Society: Learning and Meaning in the Digital Age (pp. 279-305). Cambridge: Cambridge University Press.

Clark, DB, Nelson, B, Sengupta, P, \& D’Angelo, CM. (2009). Rethinking science learning through digital games and simulations: Genres, examples, and evidence. Washington, DC: Paper commissioned for the National Research Council Workshop on Games and Simulations.

Clark, DB, Nelson, B, Chang, H, D’Angelo, CM, Slack, K, \& Martinez-Garza, M. (2011). Exploring Newtonian mechanics in a conceptually-integrated digital game: Comparison of learning and affective outcomes for students in Taiwan and the United States. Computers \& Education, 57(3), 2178-2195. doi:16/j.compedu.2011.05.007

Clark, DB, Martinez-Garza, M, Biswas, G, Luecht, RM, \& Sengupta, P. (2012). Driving assessment of students' explanations in game dialog using computer-adaptive testing and hidden Markov Modeling. In D Ifenthaler, D Eseryel, \& G Xun (Eds.), Game-based Learning: Foundations, Innovations, and Perspectives (pp. 173-199). New York: Springer.

Clark, DB, Sengupta, P, Kinnebrew, J, Killingsworth, S, Krinks, K, Martinez-Garza, M, \& Biswas, G. (2013). Enhancing Games with Assessment and Metacognitive Emphases. Washington, DC: Annual Report to the National Science Foundation.
Clark, DB, Brady, C, Sengupta, P, Martinez-Garza, M, Adams, D, Killingsworth, S, \& Van Eaton, G. (2014a). Evolving and balancing informal and formal representations. Proceedings of the Eleventh International Conference of the Learning Sciences. Boulder, CO: ISLS

Clark, DB, Tanner-Smith, E, \& Killingsworth, S. (2014). Digital games, design, and learning: A systematic review and meta-analysis. Report to The Bill and Melinda Gates Foundation. Available at http://www.sri.com/work/projects/ glasslab-research.

Collins, A. (2011). A study of expert theory formation: The role of model types and domain frameworks. In MS Khine \& I Saleh (Eds.), Models and Modeling: Cognitive Tools for Scientific Enquiry (pp. 23-40). London: Springer.

Collins, A, \& Ferguson, W. (1993). Epistemic forms and epistemic games. Educational Psychologist, 28, 25-42.

Council, NR. (2009). National Research Council Workshop on Games and Simulations. Washington, DC: National Academy Press.

Crawford, C. (1984). The Art of Computer Game Design. Berkeley, CA: Osborne/ McGraw-Hill.

Csikszentmihalyi, M. (1991). Flow: The Psychology of Optimal Experience. New York: Harper Perennial.

D'Angelo, CM, Clark, DB, Nelson, BC, Slack, K, \& Menekse, M. (2010). Connecting tacit understanding from video games to formalized vector concepts. Philadelphia, Pennsylvania: National Association of Research in Science Teaching (NARST) 2010 meeting.

Dickes, AC, \& Sengupta, P. (2013). Learning natural selection in 4th grade with multi-agent-based computational models. Research in Science Education, 43(3), 921-953.

diSessa, AA. (1983). Phenomenology and the evolution of intuition. In D Gentner \& AL Stevens (Eds.), Mental Models (pp. 15-33). Hillsdale, NJ: Lawrence Erlbaum Associates.

diSessa, AA. (1988). Knowledge in pieces. In G Forman \& P Pufall (Eds.), Constructivism in the Computer Age (pp. 49-70). Hillsdale, NJ: Lawrence Erlbaum Associates.

diSessa, AA. (1993). Toward an epistemology of physics. Cognition and Instruction, $10(2 \& 3), 105-225$.

diSessa, AA, Gillespie, NM, \& Esterly, JB. (2004). Coherence versus fragmentation in the development of the concept of force. Cognitive Science, 28(6), 843-900.

Duschl, RA, Schweingruber, HA, \& Shouse, AW. (2007). Taking Science to School: Learning and Teaching Science in Grades K-8 (National Research Council Board on Science Education, Center for Education, Division of Behavioral and Social Sciences and Education). Washington, DC: The National Academies Press.

Enyedy, N. (2005). Inventing mapping: creating cultural forms to solve collective problems. Cognition and Instruction, 23(4), 427-466.

Federation of American Scientists. (2006). Report: Summit on Educational Games: Harnessing the Power of Video Games for Learning. Washington, D.C: Federation of American Scientists.

Fox-Keller, E. (1983). A Feeling for the Organism: The Life and Work of Barbara McClintock. New York: W.H. Freeman

Garris, R, Ahlers, R, \& Driskell, JE. (2002). Games, motivation, and learning: A research and practice model. Simulation \& Gaming, 33(4), 441-467. doi:10.1177/1046878102238607.

Gee, JP. (1990). Social Linguistics and Literacies: Ideology in Discourses. London: Falmer Press.

Gee, JP. (2007a). Good Video Games and Good Learning: Collected Essays on Video Games, Learning, and Literacy (New Literacies and Digital Epistemologies). New York: Peter Lang Pub Inc.

Gee, JP. (2007b). What Video Games Have to Teach us About Learning and Literacy. Second Edition: Revised and Updated Edition (2nd ed.). New York: Palgrave Macmillan.

Gee, JP. (2008). Learning and games. In K Salen (Ed.), The Ecology of Games: Connecting Youth, Games, and Learning. The John D. and Catherine T. MacArthur Foundation Series on Digital Media and Learning (pp. 21-40). Cambridge, MA: The MIT Press. doi:10.1162/dmal.9780262693646.021.

Giere, RN. (1988). Explaining Science: A Cognitive Approach. Chicago: University of Chicago Press.

Gravemeijer, K, Cobb, P, Bowers, J, \& Whitenack, J. (2000). Symbolizing, modeling, and instructional design. In P Cobb, E Yackel, \& K McClain (Eds.), Symbolizing and Communicating in Mathematics Classrooms: Perspectives on Discourse, Tools, and Instructional Design. Mahwah, NJ: Lawrence Erlbaum Associates. 
Habgood, MPJ, \& Ainsworth, SE. (2011). Motivating children to learn effectively: exploring the value of intrinsic integration in educational games. The Journal of the Learning Sciences, 20, 169-206.

Hall, R, \& Stevens, R. (1995). Making space: A comparison of mathematical work in school and professional design practices. In SL Star (Ed.), The Cultures of Computing (pp. 118-145). London, UK: Basil Blackwell.

Hammer, D. (1996). Misconceptions or p-prims: How may alternative perspectives of cognitive structure influence instructional perceptions and intentions? The Journal of the Learning Sciences, 5(2), 97-127.

Hammer, D, Redish, EF, Elby, A, \& Scherr, RE. (2005). Resources, framing, and transfer. In J Mestre (Ed.), Transfer of Learning: Research and Perspectives (pp. 89-120). Greenwich, CT: Information Age Publishing Inc.

Hegedus, SJ, \& Moreno-Armella, L. (2009). Intersecting representation and communication infrastructures. ZDM, 41(4), 399-412.

Hegedus, S, \& Roschelle, J (Eds.). (2013). The SimCalc Vision and Contributions: Democratizing Access to Important Mathematics. New York, NY: Springer.

Hesse, MB. (1974). The Structure of Scientific Inference. Berkeley, CA: University of California Press.

Hestenes, D. (1992). Modeling games in the Newtonian world. American Journal of Physics, 60, 732-748.

Hestenes, D. (1993). MODELING is the Name of the Game. Washington DC: Presentation at the NSF Modeling Conference.

Hestenes, D, \& Halloun, I. (1995). Interpreting the force concept inventory: A response to March 1995 critique by Huffman and Heller. The Physics Teacher, 33(8), 502-506.

Hestenes, D, Wells, M, \& Swackhamer, G. (1992). Force concept inventory. The Physics Teacher, 30(3), 141-158.

Hines, PJ, Jasny, BR, \& Merris, J. (2009). Adding a T to the three R's. Science, 323, 53.

Honey, MA, \& Hilton, M (Eds.). (2010). Learning Science through Computer Games and Simulations. National Research Council. Washington, DC: National Academy Press.

Jeppsson, F, Haglund, J, Amin, TG, \& Strömdahl, H. (2013). Exploring the use of conceptual metaphors in solving problems on entropy. Journal of the Learning Sciences, 22(1), 70-120.

Kaput, J. (1994). Democratizing access to calculus: New routes to old roots. In A Schoenfeld (Ed.), Mathematical Thinking and Problem Solving (pp. 77-156). Hillsdale, NJ: Lawrence Erlbaum Associates.

Kearney, M. (2004). Classroom use of multimedia-supported predict-observeexplain tasks in a social constructivist learning environment. Research in Science Education, 34(4), 427-453.

Kiili, K. (2005). On Educational Game Design: Building Blocks of Flow Experience. Tampere, Finland: Tampere University of Technology Press.

Kiili, K, \& Lainema, T. (2008). Foundation for measuring engagement in education games. Journal of Interactive Learning Research, 19(3), 469-488.

Kirsh, D, \& Maglio, P. (1994). On the distinguishing epistemic from pragmatic action. Cognitive Science, 18, 513-549.

Koster, R. (2004). A Theory of Fun for Game Design. Scottsdale, AZ: Paraglyph Press.

Krinks, KD, Sengupta, P, \& Clark, DB. (2013). Conceptual Change in Physics through use of Digital Games. Paper presented at annual conference of the National Association for Research in Science Teaching. Puerto Rico: Rio Mar.

Latour, B. (1990). Drawing things together. In M Lynch \& S Woolgar (Eds.) Representation in Scientific Practice (pp. 19-68). Cambridge, MA: MIT Press.

Lehrer, R. (2003). Developing understanding of measurement. In J Kilpatrick, WG Martin, \& DE Schifter (Eds.), A research companion to principles and standards for school mathematics (pp. 179-192). Reston, VA: National Council of Teachers of Mathematics.

Lehrer, R. (2009). Designing to develop disciplinary dispositions: Modeling natural systems. American Psychologist, 64(8), 759-71. doi:10.1037/0003-066X.64.8.759.

Lehrer, R, \& Pritchard, C. (2002). Symbolizing space into being. In K Gravemeijer, R Lehrer, B van Oers, \& L Verschaffel (Eds.), Symbolization, Modeling and Tool use in Mathematics Education (pp. 59-86). Dordrecht, Netherlands: Kluwer Academic Press.

Lehrer, R, \& Schauble, L. (2002). Symbolic communication in mathematics and science: Co-constituting inscription and thought. In E Amsel \& J Byrnes (Eds.), The development of symbolic communication. Mahwah, NJ: Lawrence Erlbaum Associates.

Lehrer, R, \& Schauble, L. (2006a). Cultivating model-based reasoning in science education. In RK Sawyer (Ed.), The Cambridge Handbook of the Learning Sciences (pp. 371-388). Cambridge, England: Cambridge University Press.

Lehrer, R, \& Schauble, L. (2006b). Scientific thinking and science literacy: supporting development in learning in contexts. In W Damon, RM Lerner, KA
Renninger, \& IE Sigel (Eds.), Handbook of Child Psychology (6th ed., Vol. 4). Hoboken, NJ: John Wiley and Sons.

Lehrer, R, \& Schauble, L. (2010). What kind of explanation is a model? In MK Stein (Ed.), Instructional Explanations in the Disciplines (pp. 9-22). New York: Springer.

Lehrer, R, Schauble, L, Carpenter, S, \& Penner, D. (2000). The inter-related development of inscriptions and conceptual understanding. In P Cobb, E Yackel, \& K McClain (Eds.), Symbolizing and Communicating in Mathematics Classrooms: Perspectives on Discourse, Tools and Instructional Design (pp. 325-360). Mahwah, NJ: Lawrence Erlbaum Associates.

Lynch, M. (1990). The externalized retina: Selection and mathematization in the visual documentation of objects in the life sciences. In M Lynch \& S Woolgar (Eds.), Representation in Scientific Practice (pp. 153-186). Cambridge, MA: MIT Press.

Martinez-Garza, M, Clark, DB, \& Nelson, B. (2013). Digital games and the US National Research Council's science proficiency goals. Studies in Science Education, 49, 170-208. doi:10.1080/03057267.2013.839372.

Mayer, RE. (2009). Multimedia Learning (2nd ed.). New York, NY: Cambridge University Press.

Mayer, RE, \& Johnson, Cl. (2010). Adding instructional features that promote learning in a game-like environment. Journal of Educational Computing Research, 42(3), 241-265.

McGonigal, J. (2011). Reality is Broken: Why Games Make Us Better and How They Can Change the World. New York: Penguin Press.

Minstrell, J. (2001). Facets of students' thinking: Designing to cross the gap from research to standards-based practice. In K Crowley, CD Schunn, \& T Okada (Eds.), Designing for Science: Implications for Professional, Instructional, and Everyday Science. Mahwah: Lawrence Erlbaum Associates.

Morrison, D, \& Collins, A. (1995). Epistemic fluency and constructivist learning environments. Educational Technology, 35(5), 39-45.

Munz, U, Schumm, P, Wiesebrock, A, \& Allgower, F. (2007). Motivation and learning progress through educational games. Industrial Electronics, IEEE Transactions on, 54(6), 3141-3144. doi: 10.1109/TIE.2007.907030.

Nersessian, NJ. (1998). Modeling practices in conceptual change in science. In T Borsche, J Kreuzer, \& C Strub (Eds.), Cognition and Imagination (pp. 149-168). Munich: Fink Verlag.

Nersessian, NJ. (2002). The cognitive basis of model-based reasoning in science. In P Carruthers, S Stich, \& M Siegal (Eds.), The Cognitive Basis of Science (pp. 133-155). Cambridge: Cambridge University Press.

Nersessian, NJ. (2008). Creating Scientific Concepts. Cambridge, MA: MIT Press.

Pelletier, C. (2008). Gaming in context: How young people construct their gendered identities in playing and making games. In YB Kafai, C Heeter, J Denner, \& JY Sun (Eds.), Beyond Barbie and Mortal Kombat: New Perspectives on Gender and Gaming. Cambridge, Mass: The MIT Press.

Pickering, A. (1995). The Mangle of Practice: Time, Agency, and Science. Chicago: University of Chicago Press.

Rapp, DN, \& Sengupta, P. (2012). Models and modeling in science learning. Encyclopedia of the Sciences of Learning (pp. 2320-2322). New York: Springer.

Rochelle, J, \& Kaput, J. (1996). Educational software architecture and systemic impact: The promise of component software. Journal of Educational Computing Research, 14(3), 217-228.

Roy, M, \& Chi, MTH. (2005). The self-explanation principle in multimedia learning. In RE Mayer (Ed.), The Cambridge Handbook of Multimedia Learning (pp. 271-286). New York: Cambridge University Press.

Rueda, MR, Fan, J, McCandliss, BD, Halparin, JD, Gruber, DB, Lercari, LP, \& Posner, MI. (2004). Development of attentional networks in childhood. Neuropsychologia 42(8), 1029-1040. doi:10.1016/j.neuropsychologia.2003.12.012.

Salen, K, \& Zimmerman, E. (2004). Rules of Play: Game Design Fundamentals. Cambridge: MIT Press.

Schwartz, D, \& Arena, D. (2013). Measuring What Matters Most: Choice-Based Assessments for the Digital Age. Cambridge: MIT Press.

Scott, PH, Asoko, HM, \& Driver, RH. (1992). Teaching for conceptual change: a review of strategies. In R Duit, F Goldberg, \& H Niedderer (Eds.), Research in Physics Learning: Theoretical Issues and Empirical Studies (pp. 310-329). Kiel, Germany: Institute for Science Education at the University of Kiel

Sengupta, P, Clark, D, Krinks, K, Killingsworth, S, \& Brady, C. (2014). Integrating Modeling with Games for Learning Newtonian Mechanics. In N. Holbert \& D. Weintrop (Org), N. Holbert (Chair), and Y. Kafai (Discussant), Combining Video Games and Constructionist Design to Support Deep Learning in Play. In J. Poleman, E. Kyza, I. Tabak \& K. O'Neill (Eds.), Proceedings of "Learning and 
Becoming in Practice," the 11th International Conference of the Learning Sciences (ICLS 2014), pp 1388 - 1395. University of Colorado at Boulder: ISLS.

Sherin, BL. (2001). How students understand physics equations. Cognition and Instruction, 19(4), 479-541.

Slack, K, Nelson, B, Clark, DB, \& Martinez-Garza, M. (2010). Influence of Visual Cues on Learning and In-Game Performance in an Educational Physics Game Environment. Anaheim, California: Paper presented at the Association for Educational Communications and Technology (AECT) 2010 meeting.

Slack, K, Nelson, B, Clark, DB, \& Martinez-Garza, M. (2011). Model-Based Thinking in the Scaffolding Understanding by Redesigning Games for Education (SURGE) Project. New Orleans, LA: Poster presented at the American Educational Research Association (AERA) 2011 meeting.

Smith, JP, diSessa, A, \& Roschelle, J. (1993). Misconceptions reconceived: A constructivist analysis of knowledge in transition. Journal of the Learning Sciences, 3(2), 115-163.

Squire, K. (2005). Changing the game: What happens when video games enter the classroom. Innovate, 1(6), 25-49.

Squire, K. (2006). From content to context: Videogames as designed experience. Educational Researcher, 35(8), 19-29.

Squire, K. (2011). Video Games and Learning: Teaching and Participatory Culture in the Digital age. New York: Teachers College Press.

Steinkuehler, D, \& Duncan, S. (2008). Scientific habits of mind in virtual worlds. Journal of Science Education and Technology, 17(6), 530-543.

Tao, P-K, \& Gunstone, RF. (1999). The process of conceptual change in force and motion during computer-supported physics instruction. Journal of Research in Science Teaching, 36(7), 859-882.

Team, G-t-T. (2003). Design principles of next-generation digital gaming for education. Educational Technology, 43(5), 17-33.

Van Eaton, G, Clark, DB, \& Beutel, D. (2013). Designing Digital Objects to Scaffold Learning. Memphis TN: Paper presented at the 16th International Conference on Artificial Intelligence in Education (AIED 2013).

White, B. (1984). Designing computer games to help physics students understand Newton's laws of motion. Cognition and Instruction, 1, 69-108.

White, B, \& Frederiksen, J. (1998). Inquiry, modeling, and metacognition: Making science accessible to all students. Cognition and Instruction, 16(1), 3-118.

White, BY, \& Frederiksen, JR. (2000). Metacognitive facilitation: An approach to making scientific inquiry accessible to all. In JA Minstrell \& EH Van Zee (Eds.), Inquiring into Inquiry Learning and Teaching in Science (pp. 331-370). Washington, DC: American Association for the Advancement of Science.

Wilensky, U, \& Reisman, K. (2006). Thinking like a wolf, a sheep, or a firefly: Learning biology through constructing and testing computational theoriesan embodied modeling approach. Cognition and Instruction, 24(2), 171-209.

Wouters, P, van Nimwegen, C, van Oostendorp, H, \& van der Spek, ED. (2013). A meta-analysis of the cognitive and motivational effects of serious games. Journal of Educational Psychology, 105, 249-265. Doi: 10.1037/a0031311.

Wright, W. (2006). Dream machines. Wired, 14(4), 110-112.

\section{Submit your manuscript to a SpringerOpen ${ }^{\circ}$ journal and benefit from:}

- Convenient online submission

- Rigorous peer review

- Immediate publication on acceptance

- Open access: articles freely available online

- High visibility within the field

- Retaining the copyright to your article

Submit your next manuscript at $\gg$ springeropen.com 